ARTICLE

\title{
Caffeine blocks SREBP2-induced hepatic PCSK9 expression to enhance LDLR-mediated cholesterol clearance
}

\author{
Paul F. Lebeau ${ }^{1,13}$, Jae Hyun Byun ${ }^{1,13}$, Khrystyna Platko1, Paul Saliba (1) 2, Matthew Sguazzin², \\ Melissa E. MacDonald ${ }^{1}$, Guillaume Paré (10 ${ }^{3,4,5}$, Gregory R. Steinberg (10 2,6, Luke J. Janssen', \\ Suleiman A. Igdoura ${ }^{8}$, Mark A. Tarnopolsky (10) 9,10 , S. R. Wayne Chen ${ }^{11}$, Nabil G. Seidah ${ }^{12}$, Jakob Magolan (1) ${ }^{2}$ \& \\ Richard C. Austin (1) 1,5凶
}

Evidence suggests that caffeine (CF) reduces cardiovascular disease (CVD) risk. However, the mechanism by which this occurs has not yet been uncovered. Here, we investigated the effect of $\mathrm{CF}$ on the expression of two bona fide regulators of circulating low-density lipoprotein cholesterol (LDLC) levels; the proprotein convertase subtilisin/kexin type 9 (PCSK9) and the low-density lipoprotein receptor (LDLR). Following the observation that $C F$ reduced circulating PCSK9 levels and increased hepatic LDLR expression, additional CF-derived analogs with increased potency for PCSK9 inhibition compared to CF itself were developed. The PCSK9-lowering effect of CF was subsequently confirmed in a cohort of healthy volunteers. Mechanistically, we demonstrate that CF increases hepatic endoplasmic reticulum (ER) $\mathrm{Ca}^{2+}$ levels to block transcriptional activation of the sterol regulatory elementbinding protein 2 (SREBP2) responsible for the regulation of PCSK9, thereby increasing the expression of the LDLR and clearance of LDLc. Our findings highlight ER $\mathrm{Ca}^{2+}$ as a master regulator of cholesterol metabolism and identify a mechanism by which CF may protect against CVD.

\footnotetext{
${ }^{1}$ Department of Medicine, Division of Nephrology, McMaster University, The Research Institute of St. Joe's Hamilton and the Hamilton Center for Kidney Research, Hamilton, ON L8N 4A6, Canada. ${ }^{2}$ Department of Biochemistry and Biomedical Sciences, McMaster University, Hamilton, ON L8S 4L8, Canada. ${ }^{3}$ Population Health Research Institute, McMaster University, Hamilton, ON L8L 2X2, Canada. ${ }^{4}$ The Departments of Medicine, Epidemiology and Pathology, McMaster University, Hamilton, ON L8L 2X2, Canada. ${ }^{5}$ The Thrombosis and Atherosclerosis Research Institute (TaARI), Department of Medicine, David Braley Research Institute, McMaster University, Hamilton L8L 2X2, Canada. ${ }^{6}$ Centre for Metabolism, Obesity and Diabetes Research, Department of Medicine, McMaster University, Hamilton, ON L8S 4L8, Canada. ${ }^{7}$ Firestone Institute for Respiratory Health, St. Joseph's Hospital, Hamilton, ON L8S 4K1, Canada. ${ }^{8}$ Department of Biology and Pathology, McMaster University, Hamilton, ON L8S 4K1, Canada. ${ }^{9}$ Department of Medicine/Neurology, McMaster University, Hamilton, ON L8N 3Z5, Canada. ${ }^{10}$ Department of Pediatrics, McMaster University, Hamilton, ON L8S 4K1, Canada. ${ }^{11}$ Libin Cardiovascular Institute of Alberta, Department of Physiology and Pharmacology, University of Calgary, Calgary, AB T2N 2T9, Canada. ${ }^{12}$ Laboratory of Biochemical Neuroendocrinology, Clinical Research Institute of Montreal, affiliated to the University of Montreal, Montreal, QC H2W 1R7, Canada. ${ }^{13}$ These authors

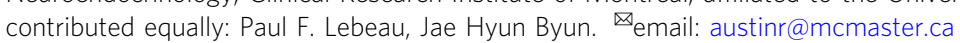


ncreased levels of circulating low-density lipoprotein cholesterol (LDLc) are tightly linked to the development of cardiovascular disease (CVD). Despite the approval of several therapies that lower LDLc, many patients fail to reach their LDL lowering goal due to intolerance, adverse events, or simply the high cost of medications. An important regulator of LDLc is the sterol regulatory element-binding protein 2 (SREBP2), which is an endoplasmic reticulum (ER)-resident transcription factor. SREBP2 is activated by reductions in intracellular cholesterol and loss of $\mathrm{ER} \mathrm{Ca}^{2+}$, which then triggers translocation to the nucleus and the induction of cholesterol regulatory genes including the proprotein convertase subtilisin/kexin type 9 (PCSK9), the lowdensity lipoprotein receptor $(L D L R)$, and HMG-CoA reductase $(H M G R)^{1}$. Recent advancements in therapies available for the management of dyslipidemia and CVD have led to the characterization of PCSK9 as a hepatocyte-secreted circulating factor capable of enhancing the degradation of cell-surface LDLR ${ }^{2-5}$. By extension, PCSK9 also reduces the ability of metabolically active tissues, such as the liver, to remove excess LDLc from the blood. Based on these seminal discoveries, anti-PCSK9 antibodies are now available to patients at high risk of CVD, yielding an unprecedented $60-70 \%$ reduction of LDLc levels ${ }^{6}$. Although efficacious, the high cost and/or need for subcutaneous administration of anti-PCSK9 antibodies poses a limit to their availability to patients worldwide ${ }^{7}$. Such circumstances warrant the need for additional studies examining the molecular mechanisms that modulate the expression and secretion of PCSK9 from hepatocytes in order to develop more cost-effective therapies.

Caffeine (CF) or 1,3,7 trimethylxanthine, is best known as a stimulant alkaloid of the central nervous system found in various plants and is commonly found in coffee and tea. The majority of published literature demonstrates that the average adult habitual caffeine drinker consumes between 400 and $600 \mathrm{mg}$ of CF daily and organizations like Health Canada and the Food and Drug Administration conclude that such doses are not negatively associated with toxicity, cardiovascular effects, bone status, calcium imbalance, behavior, the incidence of cancer or effects on male fertility ${ }^{8}$. On the contrary, accumulating evidence now suggests that moderate to high levels of CF (>600 mg), consumed daily in the form of non-alcoholic beverages, are associated with a reduction in $\mathrm{CVD}$ risk $^{8,9}$. Although biochemical studies have shown that CF increases intracellular $\mathrm{Ca}^{2+}$ levels and induces vasodilation of the vascular endothelium via release of nitric oxide $^{10,11}$, a cellular process known to be cardioprotective ${ }^{12}$, molecular mechanisms supporting clinical evidence are currently lacking.

In the current study, we demonstrate that clinically relevant concentrations of caffeine suppress SREBP2 transcriptional activation in liver hepatocytes, thereby leading to a reduction of PCSK9 in both mice and humans. Using structure/activity relationships (SAR), we have also generated several xanthine derivatives with heightened antagonism against SREBP2 and PCSK9, compared to CF. Overall, these studies characterize the mechanism by which CF impacts the expression of genes wellknown to mediate CVD risk.

\section{Results}

CF blocks PCSK9 expression and secretion in hepatocytes. To initiate our studies, cultured immortalized hepatocytes known to express and secrete PCSK9 ${ }^{13}$, including $\mathrm{HuH} 7$ and HepG2 cells, as well as primary mouse- and human-hepatocytes (PMH and $\mathrm{PHH}$, respectively), were treated with $\mathrm{CF}$ for $24 \mathrm{~h}$ and assessed for PCSK9 expression via immunoblots and real-time PCR (Fig. 1A-D). These initial experiments revealed that CF reduced protein and mRNA transcript levels of PCSK9. CF also attenuated PCSK9 expression resulting from the SERCA pump antagonist and established ER stress-inducing agent, thapsigargin (TG; Fig. $1 \mathrm{~A}, \mathrm{~B})^{13}$. Given that sterol deprivation represents another well-established promoter of SREBP2 activation ${ }^{13}$, cells were also treated with CF in the presence and absence of U18666A (U18), a pharmacological agent that depletes intracellular sterols. Like TGtreated cells, CF attenuated U18-induced PCSK9 expression (Fig. 1A; see Supplementary Table 1 in supplemental materials for a list of compounds and mechanisms of action). Importantly, CF also blocked the secretion of PCSK9 from HuH7 and HepG2 cultured hepatocytes, as well as from PMH and PHH (Fig. 1E-G). Control experiments demonstrated that CF did not interfere with the ELISA by measuring levels of recombinant PCSK9 in the presence or absence of CF (Fig. S1). A Coomassie stain of electrophoretically resolved media harvested from these cells was also used to confirm that CF was not affecting global protein secretion (Fig. 1H). Next, HepG2 cells were treated with an increasing dose of CF. Results from this experiment demonstrate that PCSK9 elicited a dynamic response to CF from the $10^{2}$ to the $10^{8} \mathrm{nM}$ range in hepatocytes (Fig. 1I). To determine whether caffeine was affecting PCSK9 expression and secretion in a transcriptional or post-transcriptional manner, HepG2 cells were pre-treated with a transcription blocker (actinomycin $\mathrm{D}[\mathrm{ActD}]$ ) and subsequently exposed to CF. The failure of CF to block PCSK9 mRNA (Fig. 1J) and secreted protein (Fig. $1 \mathrm{~K}$ ) in the presence of ActD suggests that CF exerts its effect on PCSK9 in a transcription-dependent manner. In support of these findings, CF also failed to block the secretion of PCSK9 in cells transfected with a CMV-driven PCSK9 vector (Fig. 1L). These data demonstrate that CF, in the nanomolar range, can cause a significant reduction of PCSK9 expression at the mRNA and protein levels in a variety of cultured hepatocyte cell models and that the inhibition likely occurs at the transcriptional level.

CF blocks SREBP2 activation in hepatocytes. Our research group has previously demonstrated that ER stress, specifically resulting from the depletion of $\mathrm{ER} \mathrm{Ca}^{2+}$, promotes the activation of SREBP2 and the expression of PCSK9 ${ }^{13-15}$. We, therefore, examined the effect of CF on TG-induced SREBP2 activation. Consistent with previous studies ${ }^{16}$, we observed that CF blocked the expression of SREBP2 in PMHs and PHHs, as well as in HepG2 cells (Fig. 2A-C). CF also blocked the expression of a downstream target of SREBP2 transcriptional activity in PMHs, HMGR (Fig. 2A), as well as SREBP1, the isoform known to regulate fatty acid synthesis (Fig. 2D). The effect of $\mathrm{CF}$ on the expression of hepatocyte nuclear factor 1a, a liver-expressed transcription factor also known to regulate PCSK9 expression ${ }^{17}$, was assessed but did not yield a significant difference in the absence of TG (Fig. S2). SREBP2 activity was then examined at the protein level in $\mathrm{HuH} 7$ cells transfected with a plasmid encoding GFP driven by the sterol regulatory element (SRE-GFP; Fig. 2E-G). Consistent with the real-time PCR data, we observed that $\mathrm{CF}$ blocked the nuclear/activated isoform of SREBP2 (nSREBP2; $\sim 60 \mathrm{kDa}$ ) and the expression of SRE-driven GFP in the presence and absence of TG. GFP expression was also visualized via immunofluorescent staining and quantified using ImageJ Software. Immunofluorescent staining of SREBP2 in cells treated with TG in the presence and absence of CF also demonstrated that CF attenuated the re-localization of SREBP2 from the perinuclear region to the nucleus (Fig. $2 \mathrm{H}$; nuclei containing activated SREBP2 are indicated by white arrows). Given the well-established role of SREBP2 in the transcriptional regulation of PCSK9, our data suggest that CF reduces PCSK9 expression and secretion by antagonizing de novo synthesis. 

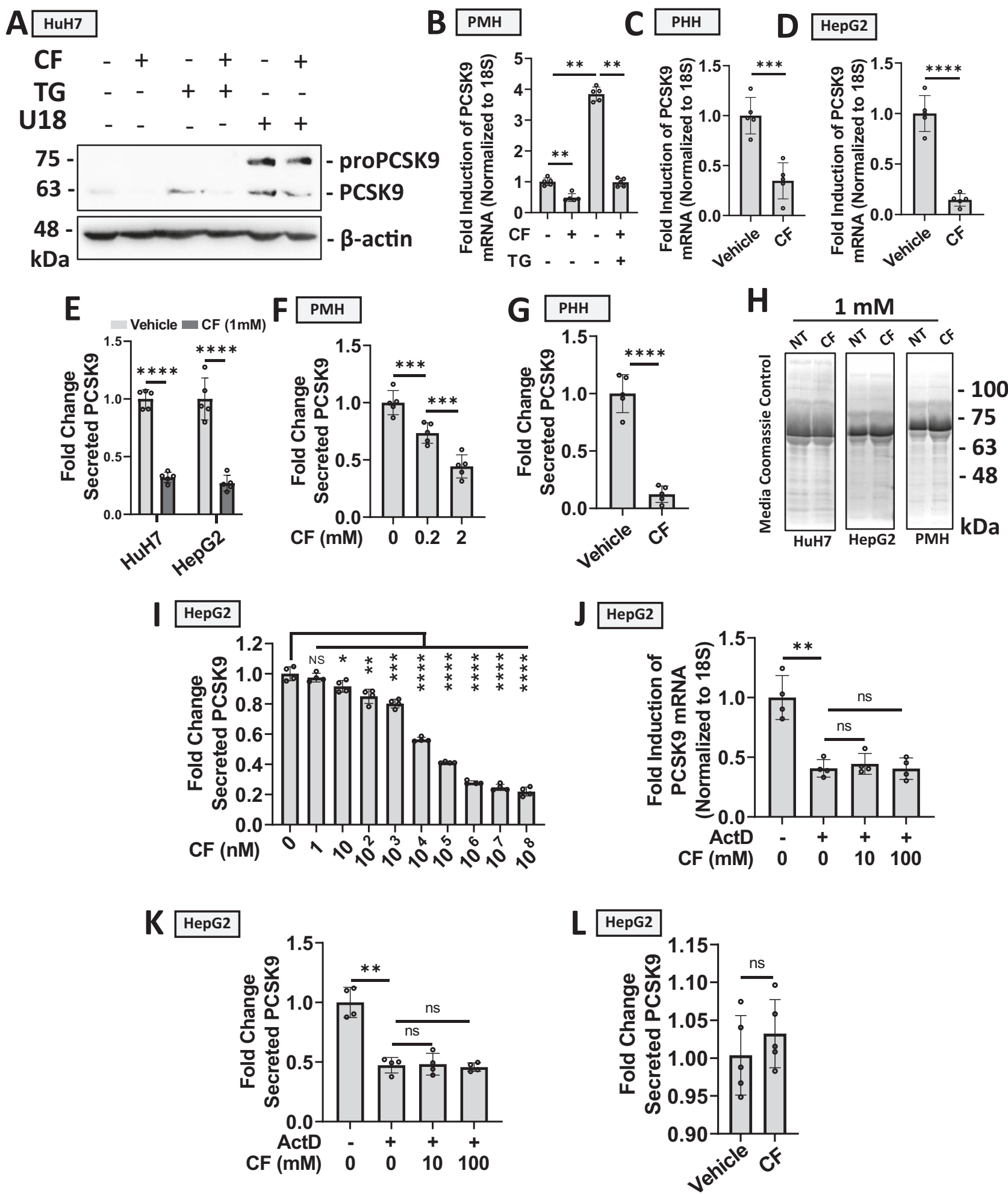

Previous studies have also shown that CF can promote the phosphorylation and activation of $\mathrm{AMPK}^{16,18}$, a liver-expressed kinase known to induce the inhibitory phosphorylation of SREBP $1 c^{19}$. Similar results were observed in our study, whereby CF treatment induced the phosphorylation and activation of AMPK (pAMPK) and subsequent induction of a downstream marker (phosphorylated acetyl CoA carboxylase [pACC]) in the livers of $\mathrm{C} 57 \mathrm{BL} / 6 \mathrm{~J}$ mice (Fig. S3A). PMHs were then isolated from wildtype (WT) and $A m p k \beta 1^{-I-}$ mice $^{20}$. Treatment of these hepatocytes with CF led to a reduction in PCSK9 expression and secretion (Fig. S3B, C), suggesting that AMPK is not directly involved in CF-mediated PCSK9 inhibition. A similar result was observed in hepatocytes treated with CDN1163 (CDN), a pharmacologic agent known to increase $\mathrm{ER} \mathrm{Ca}^{2+}$ levels by inducing SERCA pump activation (Fig. S3D, E) ${ }^{21}$.

ER $\mathrm{Ca}^{2+}$ modulates PCSK9 expression and secretion. Among the many intracellular effects of $\mathrm{CF}$, its ability to increase intracellular $\mathrm{Ca}^{2+}$ levels is well-studied ${ }^{11}$. Given our previous report 
Fig. 1 Caffeine blocks PCSK9 expression and secretion in hepatocytes. A HuH7 cells were treated with established inducers of PCSK9 expression, thapsigargin (TG; $100 \mathrm{nM})$ or U18 $(10 \mu \mathrm{M})$, in the presence or absence of caffeine (CF; $200 \mu \mathrm{M})$ for $24 \mathrm{~h}^{13}$. PCSK9 expression was assessed via immunoblot analysis. B-D PCSK9 expression was also assessed in primary mouse hepatocytes (PMH) and primary human hepatocytes (PHH), as well as in HepG2 cells treated with CF and TG via real-time PCR ( $n=5$ biologically independent samples per group; data presented are mean \pm s.d.). E-G PCSK9 ELISAs were assayed on the medium harvested from CF-treated $\mathrm{HuH7}, \mathrm{HepG} 2, \mathrm{PMHs}$, and $\mathrm{PHHs}$ ( $n=5$ biologically independent samples per group). H Coomassie blue staining of electrophoretically resolved medium harvested from CF-treated cells served to examine the effect of CF on total secreted protein levels. I Secreted PCSK9 levels from HepG2 cells treated with an increasing dose of CF ( $n=4$ biologically independent samples per group; data presented are mean \pm s.d). J-K PCSK9 expression and secretion were assessed in HepG2 cells treated in the presence and absence of CF and a blocker of transcription, ActD $(10 \mu \mathrm{M})(n=4$ biologically independent samples per group; data presented are mean \pm s.d). L Finally, ELISAs were also used to measure secreted PCSK9 levels in CF-treated HepG2 cells $(1 \mathrm{mM})$ transfected with a CMV-driven PCSK9 vector $(n=5$ biologically independent samples per group; data presented are mean \pm s.d). Statistical comparisons between two groups were conducted using unpaired two-tailed Student's t-tests, while comparisons between multiple groups were compared using one-way ANOVAs with the Tukey HSD post-hoc test $\left({ }^{\star} p<0.05 ;{ }^{\star \star} p<0.01 ;{ }^{* \star *} p<0.001\right.$; $\left.{ }^{\star \star \star \star} p<0.0001\right)$. Source data are provided as a Source Data file.

showing that $\mathrm{ER} \mathrm{Ca}^{2+}$ depletion induces SREBP2 activation ${ }^{13}$, we postulated here that (a) CF may increase ER $\mathrm{Ca}^{2+}$ levels, and (b) other agents known to increase $\mathrm{ER} \mathrm{Ca}^{2+}$ levels may also block SREBP2 activation and PCSK9 expression. To test this hypothesis, we first examined cytosolic $\mathrm{Ca}^{2+}$ levels in CF-treated cells using the high-affinity fluorescent $\mathrm{Ca}^{2+}$ indicator, Fura-2-AM. Consistent with previous studies, CF significantly increased cytosolic $\mathrm{Ca}^{2+}$ levels in immortalized hepatocytes (Fig. S4). ER $\mathrm{Ca}^{2+}$ levels were then examined in cells transfected with D1ER; a genetically encoded ER-resident fluorescence resonance energy transfer (FRET)-based calreticulin chameleon $\mathrm{Ca}^{2+}$ sensor, which increases in fluorescence intensity upon $\mathrm{Ca}^{2+}$ binding ${ }^{22}$. The lowaffinity $\mathrm{Ca}^{2+}$ indicator, Mag-Fluo-4, was also utilized for the direct assessment of $\mathrm{ER} \mathrm{Ca}^{2+}$ and increases in fluorescence intensity upon $\mathrm{Ca}^{2+}$ binding ${ }^{23,24}$. The fluorescence intensity of cells treated with $\mathrm{CF}$ and control agents, TG and CDN, was assessed using a fluorescent spectrophotometer and visualized using a fluorescent microscope (Fig. 3A). In addition to heightened cytosolic $\mathrm{Ca}^{2+}$ levels, we also observed that $\mathrm{CF}$ increased $\mathrm{ER} \mathrm{Ca}^{2+}$ levels. As expected, the control agent $\mathrm{CDN}$ increased ER $\mathrm{Ca}^{2+}$ levels, whereas TG reduced ER $\mathrm{Ca}^{2+}$ levels. ER $\mathrm{Ca}^{2+}$ content was also assessed indirectly with the high-affinity $\mathrm{Ca}^{2+}$ dye, Fura-2-AM (Fig. 3B). HuH7 cells were pretreated with CF for $24 \mathrm{~h}$ and subsequently exposed to a high dose of TG, which causes a spontaneous loss of $\mathrm{ER} \mathrm{Ca}^{2+}$. In response to this, cells pretreated with CF exhibited increased ER $\mathrm{Ca}^{2+}$ efflux compared to cells treated with the vehicle control when exposed to TG. We also observed that the protein expression of calnexin, an ERresident protein with high capacity for $\mathrm{Ca}^{2+}$ binding ${ }^{25}$, was induced by $\mathrm{CF}$ and blocked by TG (Fig. 3C).

To further test our hypothesis that increasing $\mathrm{ER} \mathrm{Ca}^{2+}$ blocks PCSK9 expression, we treated cells with a variety of wellestablished $\mathrm{Ca}^{2+}$-modulating agents. At low dose $(10 \mathrm{nM})$, ryanodine is known to facilitate $\mathrm{ER} \mathrm{Ca}^{2+}$ loss by enhancing RyR-mediated $\mathrm{Ca}^{2+}$ transients; whereas high dose ryanodine $(10 \mu \mathrm{M})$ is known to block RyR-mediated $\mathrm{ER} \mathrm{Ca}^{2+}$ leakage $^{26}$. The compound $2 \mathrm{APB}$ also blocks the exit of $\mathrm{ER} \mathrm{Ca}^{2+}$ by antagonizing IP3Rs ${ }^{27,28}$. In contrast to these two agents that modulate $\mathrm{ER} \mathrm{Ca}^{2+}$ release, $\mathrm{CDN}$ is an established allosteric activator of the SERCA pump and thus increases the entry of $\mathrm{Ca}^{2+}$ into the $\mathrm{ER}^{7}$. Consistent with our hypothesis, we observed that high-dose ryanodine, $2 \mathrm{APB}$, and CDN, blocked SREBP2 and PCSK9 at the mRNA transcript level in the presence and absence of TG (Fig. 3D- F). These agents also blocked TG-induced expression of the $\mathrm{Ca}^{2+}$-dependent chaperone and ER stress marker, GRP78. Consistent with our previous studies, we also observed that ER $\mathrm{Ca}^{2+}$ depletion via TG and cyclopiazonic acid (CPA) treatment increased PCSK9 and SREBP2 expression (Fig. 3G, H) ${ }^{13}$. As expected, these established ER stress-inducing agents also increased the expression of GRP78.
Secreted PCSK9 levels in the media harvested from cells treated with $\mathrm{Ca}^{2+}$-modulating agents were then assessed using ELISAs. Consistent with real-time PCR findings, we observed that highdose ryanodine, CDN, and 2APB reduced PCSK9 secretion (Fig. 3I). Overexpression of calnexin and loss-of function ryanodine receptor variants $\left(\mathrm{RyR}^{\mathrm{E} 4872 \mathrm{~A}}\right.$ and $\mathrm{RyR}^{\mathrm{A} 4860 \mathrm{G}}$ ), which were previously shown to increase $\mathrm{ER} \mathrm{Ca}^{2+}$ levels ${ }^{25,27}$, also blocked PCSK9 secretion (Fig. 3J, K). In contrast to its effect on PCSK9 mRNA transcript levels, we also observed that TG reduced PCSK9 secretion (Fig. 3L); an observation consistent with our previous study ${ }^{13}$. Sterol deprivation via treatment with U18, which is not known to affect $\mathrm{ER} \mathrm{Ca}^{2+}$ levels, yielded findings consistent with our previous observations ${ }^{13}$ and increased PCSK9 secretion (Fig. 3M). Finally, to confirm that CF blocked PCSK9 secretion in a $\mathrm{Ca}^{2+}$-dependent manner, experiments were repeated in HepG2 cells incubated in $\mathrm{Ca}^{2+}$. deficient medium for $48 \mathrm{~h}$ (Fig. 3N). We previously demonstrated that this treatment caused robust ER stress and likely explains the observed reduction of secreted PCSK9 levels in the absence of CF. Importantly, however, these data reveal that $\mathrm{CF}$ failed to antagonize PCSK9 secretion in cells that have been deprived of $\mathrm{Ca}^{2+}$. Overall, these data provide strong evidence that $\mathrm{ER} \mathrm{Ca}^{2+}$ levels not only affect the expression of ER stress markers, but also regulate PCSK9 and SREBP2.

\section{$\mathrm{Ca}^{2+}$ increases the binding capacity of GRP78 for ER-resident} SREBP2 and prevents its exit from the ER. GRP78 is among a number of $\mathrm{Ca}^{2+}$-dependent chaperones that play a central role in facilitating a chemical equilibrium that favors elevated $\mathrm{Ca}^{2+}$ levels in the ER lumen relative to the cytosol via direct binding/ sequestration and buffering ${ }^{29}$. It is estimated that GRP78 increases the $\mathrm{Ca}^{2+}$-retaining ability of the ER by $25 \%{ }^{30}$. Given that chaperones increase $\mathrm{ER} \mathrm{Ca}^{2+}$ levels but are also $\mathrm{Ca}^{2+}$ dependent in their capacity to bind and fold polypeptides, we investigated whether $\mathrm{ER} \mathrm{Ca}^{2+}$ could modulate the ability of GRP78 to interact with ER-resident pre-mature SREBP2 $(\sim 125 \mathrm{kDa})$. In support of this notion, previous studies have demonstrated that (a) GRP78 is highly promiscuous in its client specificity ${ }^{31}$, capable of binding to one site every 36 amino acids of a randomly generated peptide ${ }^{32}$, (b) $\mathrm{Ca}^{2+}$ and ATP bind to GRP78 in a cooperative manner and that ATP is necessary for the peptide binding and folding abilities of this chaperone ${ }^{33}$, and (c) overexpression of GRP78 can attenuate the activation of SREBPs in response to ER stress ${ }^{14}$.

To determine whether increasing ER $\mathrm{Ca}^{2+}$ levels enhance GRP78 peptide binding capacity, HuH7 cells were treated with either $\mathrm{CDN}$, which increases $\mathrm{ER} \mathrm{Ca}^{2+}$ levels, or TG which causes $\mathrm{ER} \mathrm{Ca}^{2+}$ depletion. Following treatment, the interaction between GRP78 and SREBP2 was examined via immunoprecipitation of the former. By affecting ER $\mathrm{Ca}^{2+}$ levels, however, these agents 

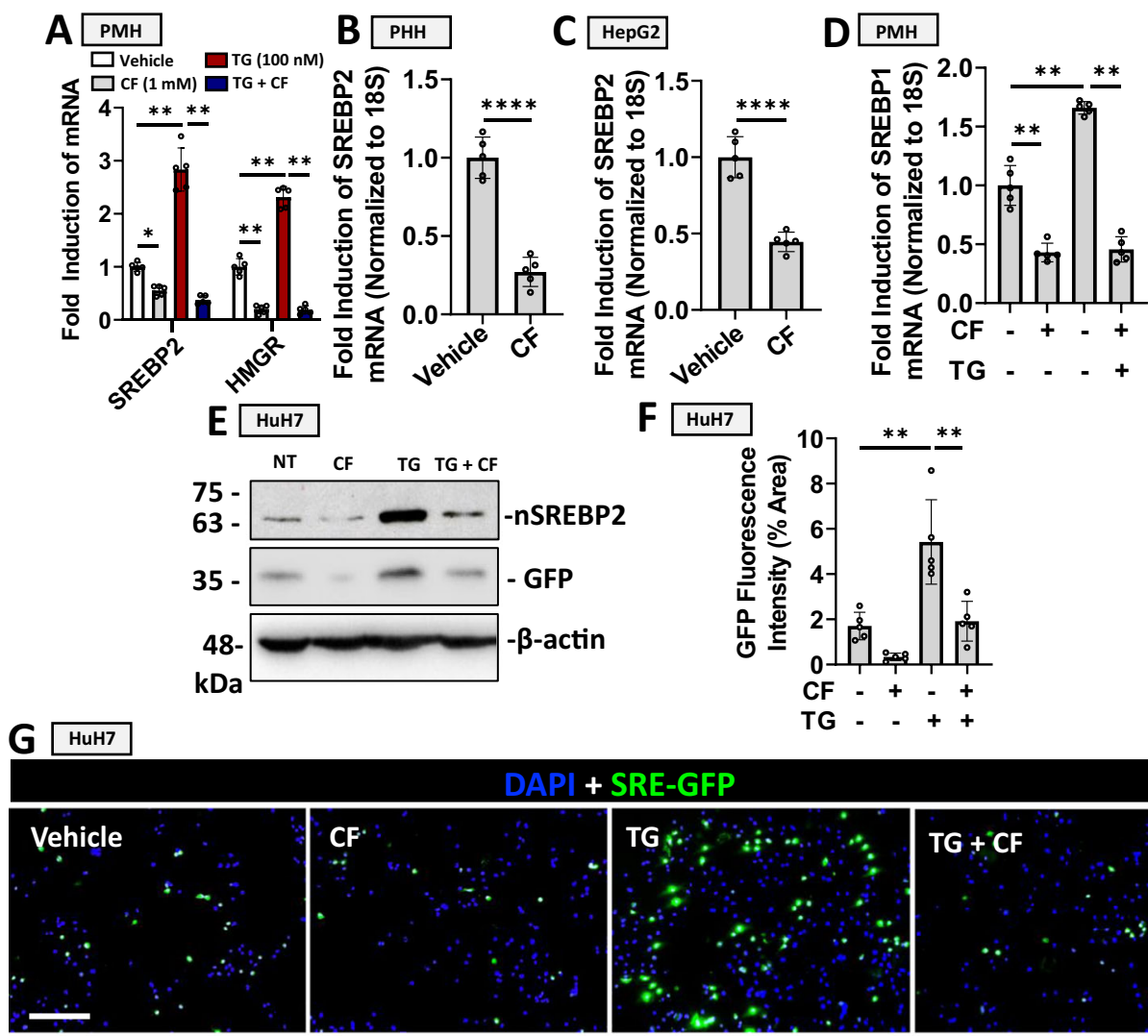

DAPI + SRE-GFP
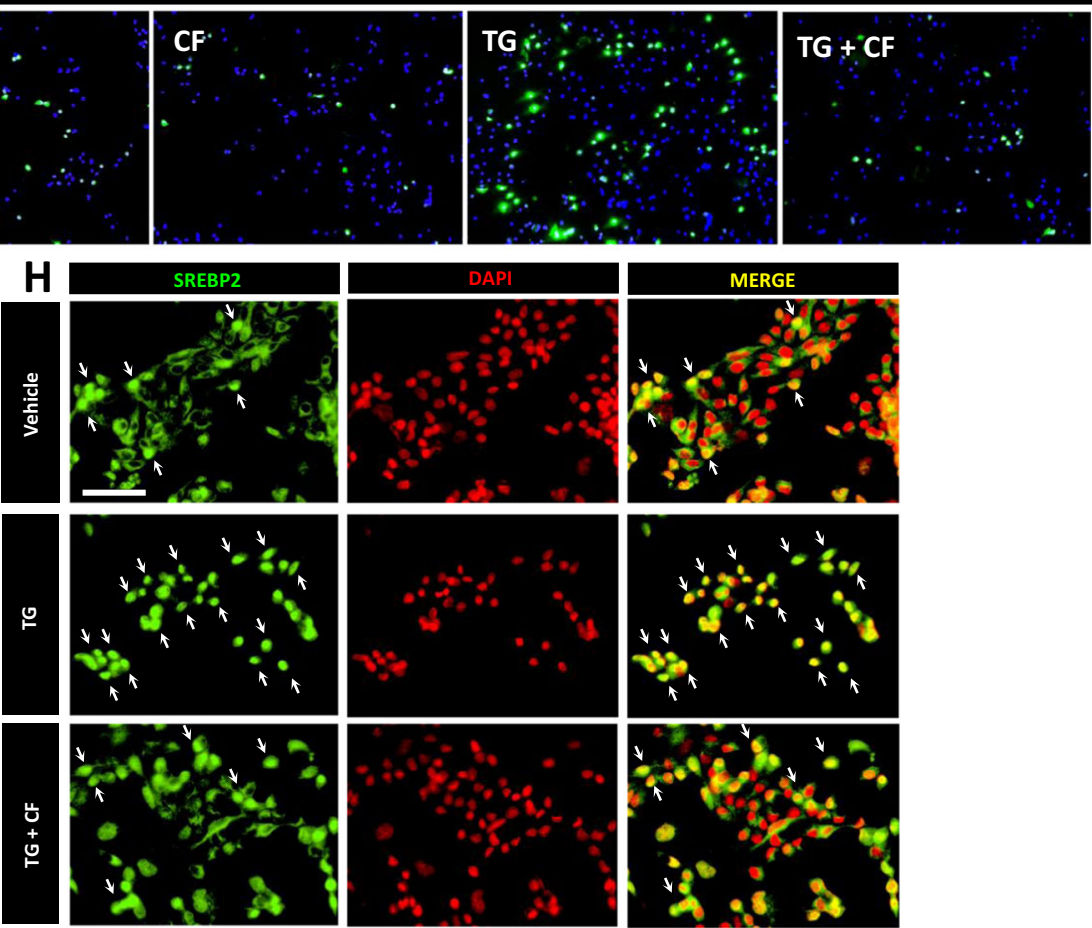

Fig. 2 Caffeine blocks SREBP2 activation in hepatocytes. A The effect of caffeine (CF; $200 \mu$ M) on SREBP2 and SREBP1 mRNA expression was examined in primary mouse hepatocytes (PMH) in the presence and absence of thapsigargin (TG; $100 \mathrm{nM}$ ), an established activator of SREBPs. The downstream product of SREBP2 transcriptional activity, HMGR, was also examined. B, C The inhibitory effect of CF on SREBP2 was also examined in primary human hepatocytes $(\mathrm{PHH})$ and HepG2 cells. D CF-mediated SREBP1 inhibition was also examined in PMH $\left({ }^{\star} p<0.05\right)$. E-G HuH7 cells were transfected with a reporter construct encoding a sterol-regulatory element-driven green fluorescent protein (SRE-GFP; green color). Cells were subsequently treated with CF $(200 \mu \mathrm{M})$ and/or TG $(100 \mathrm{nM}) 24 \mathrm{~h}$ later. GFP and nuclear (n)SREBP2 expression were examined via immunoblot analysis. GFP expression was also assessed via immunofluorescent staining, which was quantified using ImageJ. $\mathbf{H}$ The cellular localization of SREBP2 (green color) in CF- and TG-treated $\mathrm{HuH7}$ cells was also examined via immunofluorescent staining. Nuclei containing activated SREBP2 are indicated by white arrows. For all data in this figure, $n=5$ biologically independent samples per group; data presented are mean \pm s.d). Scale bars; $\mathbf{G} 100 \mu \mathrm{m} ; \mathbf{H} 20 \mu \mathrm{m}$. Statistical comparisons between two groups were conducted using unpaired two-tailed Student's $t$-tests, while comparisons between multiple groups were compared using one-way ANOVAs with the Tukey HSD post-hoc test $\left({ }^{\star} p<0.05 ;{ }^{\star \star} p<0.01 ;{ }^{\star \star \star} p<0.001 ;{ }^{\star \star \star \star} p<0.0001\right)$. Source data are provided as a Source Data file.

also directly impact the expression and abundance of GRP78 compared to untreated cells. Therefore, assessment of the relative binding capacity GRP78 for SREBP2 between treatments required normalization of immunoprecipitations to equivalent GRP78 protein levels (Fig. 4A). Following conditions of TG-induced ER
$\mathrm{Ca}^{2+}$ depletion and stress, GRP78 lost its intrinsic ability to interact with pre-mature SREBP2. Conversely, increasing ER $\mathrm{Ca}^{2+}$ levels via CDN treatment enhanced the ability of GRP78 to interact with and sequester pre-mature SREBP2. Strikingly, we observed that CF- and CDN-treated cells behaved similarly, 

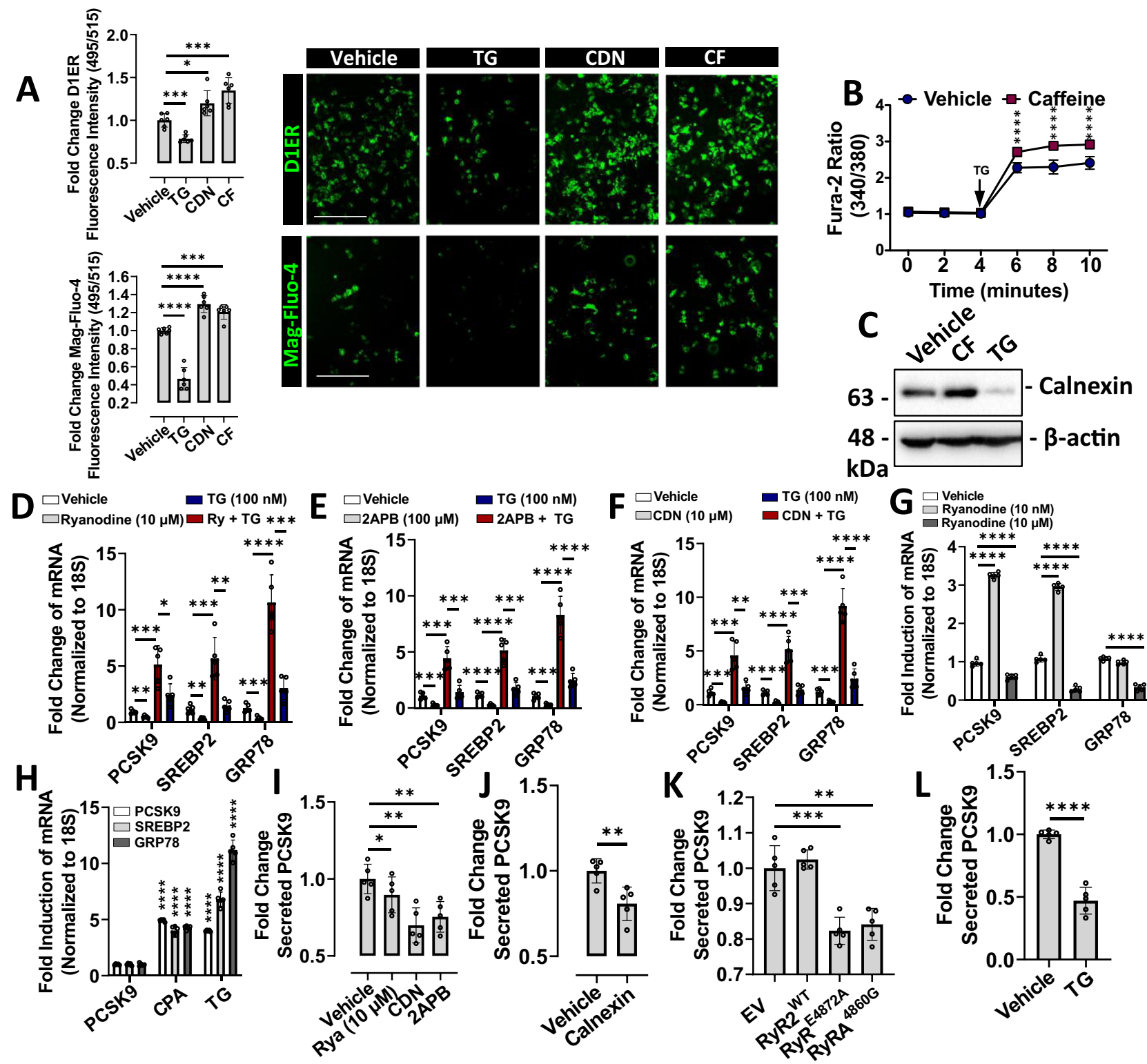

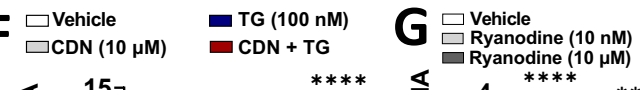
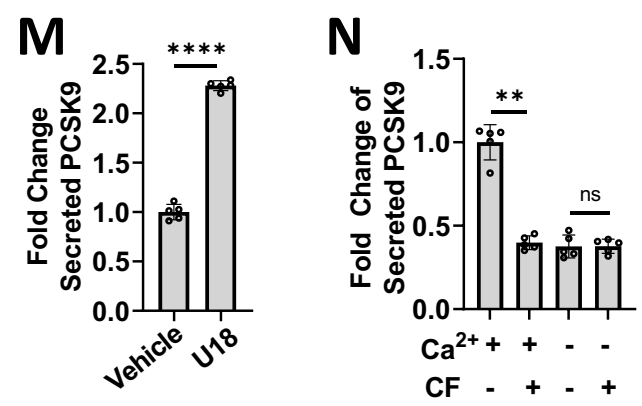

suggesting that CF also increases the SREBP2-binding capacity of GRP78. Consistent with immunoprecipitations, immunoblots of whole-cell lysates demonstrate that CF and CDN antagonized the activation and nuclear localization of SREBP2, whereas TG had the opposite effect (Fig. 4B).

To confirm that CF blocked SREBP2 activation in a manner dependent on GRP78, cells transfected with siRNA targeted against GRP78 (siGRP78) were also treated with CF. Our findings demonstrate that siGRP78 treatment significantly increased the mRNA and secreted forms of PCSK9, as well as the mRNA levels of SREBP2 (Fig. 4C, D). Consistent with these findings, we also observed that CF failed to attenuate PCSK9 and SREBP2 mRNA expression or secreted PCSK9 levels in the presence of siGRP78 (Fig. 4D, E). The siRNA-mediated knockdown of GRP78 was confirmed via immunoblotting (Fig. 4F).

Because $\mathrm{ER} \mathrm{Ca}^{2+}$ depletion induces a compensatory unfolded protein response (UPR), we also postulated that CF may attenuate UPR marker expression by increasing $\mathrm{ER} \mathrm{Ca}^{2+}$ levels. Upon assessment of PHHs treated with $\mathrm{CF}$, we observed a reduction in mRNA transcript levels of ER stress markers GRP78 
Fig. 3 Endoplasmic reticulum $\mathbf{C a}^{2}+$ modulates PCSK9 expression and secretion. A HuH7 cells were either transfected with a FRET-based ER-resident $\mathrm{Ca}^{2+}$ sensor, D1ER, or pre-loaded with the low-affinity $\mathrm{Ca}^{2+}$ indicator, Mag-Fluo-4 (green color). Cells were subsequently treated with either thapsigargin (TG; $100 \mathrm{nM}), \mathrm{CDN}(100 \mu \mathrm{M})$ or caffeine $(\mathrm{CF} ; 200 \mu \mathrm{M})$ for $24 \mathrm{~h}$. Fluorescence intensity was measured using a fluorescent spectrophotometer and visualized using a fluorescent microscope $(n=6$ biologically independent samples per group; data presented are mean $\pm \mathrm{s}$.d.). B HuH7 cells were pretreated with either CF or vehicle for $24 \mathrm{~h}$ and loaded with the high-affinity $\mathrm{Ca}^{2+}$ dye, Fura-2-AM. Exposure of cells to a high dose of TG (1 mM) induced a spontaneous depletion of endoplasmic reticulum (ER) $\mathrm{Ca}^{2+}\left({ }^{*}, p<0.05\right.$ vs. vehicle-treated). C The expression of an ER-resident $\mathrm{Ca}{ }^{2+}$ binding protein, calnexin, was examined in CF- and TG-treated HuH7 cells using immunoblots. D-G PCSK9, SREBP2, and GRP78 mRNA expression was assessed in HuH7 cells treated with a variety of ER $\mathrm{Ca}^{2+}$ modulators including: ryanodine receptor agonist (ryanodine, $10 \mathrm{nM}$ ), ryanodine receptor antagonist (ryanodine, $10 \mu \mathrm{M})$, SERCA pump activator CDN $(100 \mu \mathrm{M})$ and IP3R antagonist 2APB $(50 \mu \mathrm{m})$, in the presence and absence of TG (100 nM) for $24 \mathrm{~h}$. $\mathbf{H} \mathrm{mRNA}$ transcript levels were also examined in HuH7 cells treated with SERCA pump inhibitors, TG (100 nM), and CPA (10 $\mu$ M). I-K The effect of pharmacologic agents and plasmid-derived CMV-driven proteins, known to affect ER Ca ${ }^{2+}$ levels, on secreted PCSK9 levels was then examined using ELISAs.

L, M Secreted PCSK9 levels were also examined in TG- and U18-treated cells. $\mathbf{N}$ The effect of CF on secreted PCSK9 levels was also examined in cells incubated in $\mathrm{Ca}^{2+}$-deficient medium. For panels D-N: $n=5$ biologically independent samples per group; data presented are mean \pm s.d. Scale bars; $200 \mu$ m. Statistical comparisons between two groups were conducted using unpaired two-tailed Student's $t$-tests, while multiple groups were compared using oneway ANOVAs with the Tukey HSD post hoc test $\left({ }^{\star} p<0.05 ;{ }^{\star \star} p<0.01 ;{ }^{\star \star \star} p<0.001 ;{ }^{\star \star \star \star} p<0.0001\right)$. Source data are provided as a Source Data file.

and the activating transcription factor 4 (ATF4; Fig. 4G). Similar experiments were also carried out in cultured $\mathrm{HuH7}$ cells (Fig. S5A, B), in which a CF-mediated reduction in the expression of PPERK, IRE1 $\alpha$, sXBP1, ATF4 and ATF6 was also observed via immunoblotting and real-time PCR. Reactive oxygen species production, a process known to occur during conditions of ER stress, was also attenuated by CF (Fig. $5 \mathrm{H}$ ). Consistent with its effect on ER stress markers, CF blocked the accumulation of thioflavin-T-stained misfolded protein aggregates and the expression of FLAG-sXBP1 in cells transfected with the ER activated indicator plasmid ${ }^{34}$ (Fig. 4I-K). Collectively, these data support a model in which heightened $\mathrm{ER} \mathrm{Ca}{ }^{2+}$ levels promote chaperone function and efficiency, thereby leading to a reduction in chaperone abundance. While increasing the protein binding ability of chaperones, such as GRP78, CF also attenuates SREBP2driven gene expression (Fig. 4L).

CF blocks hepatic ER chaperone expression and attenuates PCSK9 secretion in mice. Next, we assessed the effect of CF on PCSK9 expression/secretion and UPR activation in mice. Following IP injection of CF $(50 \mathrm{mg} / \mathrm{kg}-8 \mathrm{~h})$ we observed a significant reduction of circulating PCSK9 and triglyceride levels (Fig. 5A, B). A time-course experiment also revealed that $\mathrm{CF}$ treatment required $4 \mathrm{~h}$ to significantly reduce plasma PCSK9 levels in mice (Fig. 5C). Consistent with these observations, administration of CF via oral-gavage $(20 \mathrm{mg} / \mathrm{kg})$ also reduced circulating PCSK9 levels (Fig. S6) The protein and mRNA expression of UPR chaperones, GRP78, GRP94, IRE1a, and CHOP was also assessed via immunohistochemical staining, immunoblots and real-time PCR in the livers of these mice. Consistent with our findings in cultured cells, CF reduced the expression of UPR markers (Fig. 5D-G). Likewise, in a manner similar to previous reports, an inverse correlation between plasma PCSK9 levels and the expression of hepatic cell-surface LDLR and CD36 protein was observed (Fig. 5D-E) ${ }^{2,4,35}$. LDLR staining was also performed in $P c s k 9^{-1-}$ and $\mathrm{Ldlr}^{-1-}$ mice to confirm the specificity of the antibodies used in our study (Fig. S7). Immunohistochemical staining intensities were quantified using ImageJ Software (Fig. 5E). Despite the increase in hepatic cell-surface LDLR levels, real-time PCR data revealed that LDLR transcript levels were reduced by CF; a result that is consistent with other SREBP-2 regulated genes. Similar to CF, CDN also increased hepatic LDLR expression in mice (Fig. S5C. $50 \mathrm{mg} / \mathrm{kg}$; IP; $8 \mathrm{~h}$ ).

To confirm that our mouse model was responding to treatments in a manner consistent with previous studies, mice were treated with alirocumab; a well-established clinically approved anti-PCSK9 monoclonal antibody ${ }^{36}$. Treatment with alirocumab led to a significant increase in hepatic LDLR expression (Fig. 5H, I). A reduction in the mRNA levels of SREBP2, PCSK9 and the LDLR was also observed (Fig. 5J). Overall, these studies demonstrate that CF blocks the secretion of PCSK9 and increases the expression of the LDLR in vivo.

CF increases hepatic LDL uptake. It is well-established that PCSK9 enhances the degradation of the LDLR and reduces the capacity of hepatocytes to bind and internalize extracellular $\mathrm{LDLc}^{37}$. We therefore postulated that $\mathrm{CF}$ and other agents that increase $\mathrm{ER} \mathrm{Ca}^{2+}$ levels, may also augment LDLc clearance. We started by confirming that CF increased the expression of PCSK9regulated receptors in our cultured cell models using immunoblots (Fig. 6A). Next, we developed an assay whereby HepG2 cells plated in black clear-bottom 96-well plates were treated with agents for $24 \mathrm{~h}$ and subsequently exposed to fluorescently labeled DiI-LDL for $5 \mathrm{~h}$ in FBS-free medium prior to analysis. The uptake and accumulation of DiI-LDL was then quantified using a fluorescent spectrophotometer (Molecular Devices). Interestingly, we observed that CF increased LDLc uptake and that U18, an agent that increased secreted PCSK9 levels (Fig. 3M), reduced LDLc uptake (Fig. 6B). To confirm that CF increased LDLc uptake in cultured hepatocytes in a manner dependent on PCSK9 inhibition, this experiment was repeated in HepG2 cells stably transfected with PCSK9 shRNA (Fig. 6C). As expected, CF treatment failed to significantly increase LDLc uptake in conditions of reduced PCSK9 levels. Live-cell staining of the LDLR was also performed in HepG2 cells exposed to DiI-LDL (Fig. 6D). Increased cell-surface LDLR, as well as intracellular DiI-LDL, was observed in CF-treated HepG2 cells compared to vehicle-treated cells using a fluorescent microscope. Despite the increased protein abundance of the LDLR, as well as increased uptake of LDLc, CF reduced mRNA transcript levels of the LDLR in $\mathrm{HuH7}$ and HepG2 cells (Fig. 6E). Given the observed increase in CD36 receptor levels, additional experiments were conducted to determine whether CD36 played a role in LDLc uptake in response to CF. Results from these experiments demonstrate that the knockdown of CD36 via siRNA (siCD36), as well as the pharmacologic inhibition using sulfosuccinimidyl oleate (SSO), failed to affect CF-mediated DiI-LDL uptake (Fig. 6F, G). The knockdown of CD36 was confirmed via immunoblotting (Fig. $6 \mathrm{H}$ ).

We next examined the effect of CF on hepatic LDLc uptake in mice. Accordingly, Pcsk $9^{+/+}$and Pcsk9-I- mice were treated with either CF or PBS-vehicle for $8 \mathrm{~h}$, as well as fluorescently labeled DiI-LDL for $1 \mathrm{~h}$ prior to sacrifice. In support of our in vitro studies, we observed that CF increased hepatic cellsurface LDLR expression in the Pcsk9+/+ mice but did not increase LDLR expression in Pcsk9-1- mice (Fig. 6I). CF also increased hepatic DiI fluorescence intensity and reduced serum 


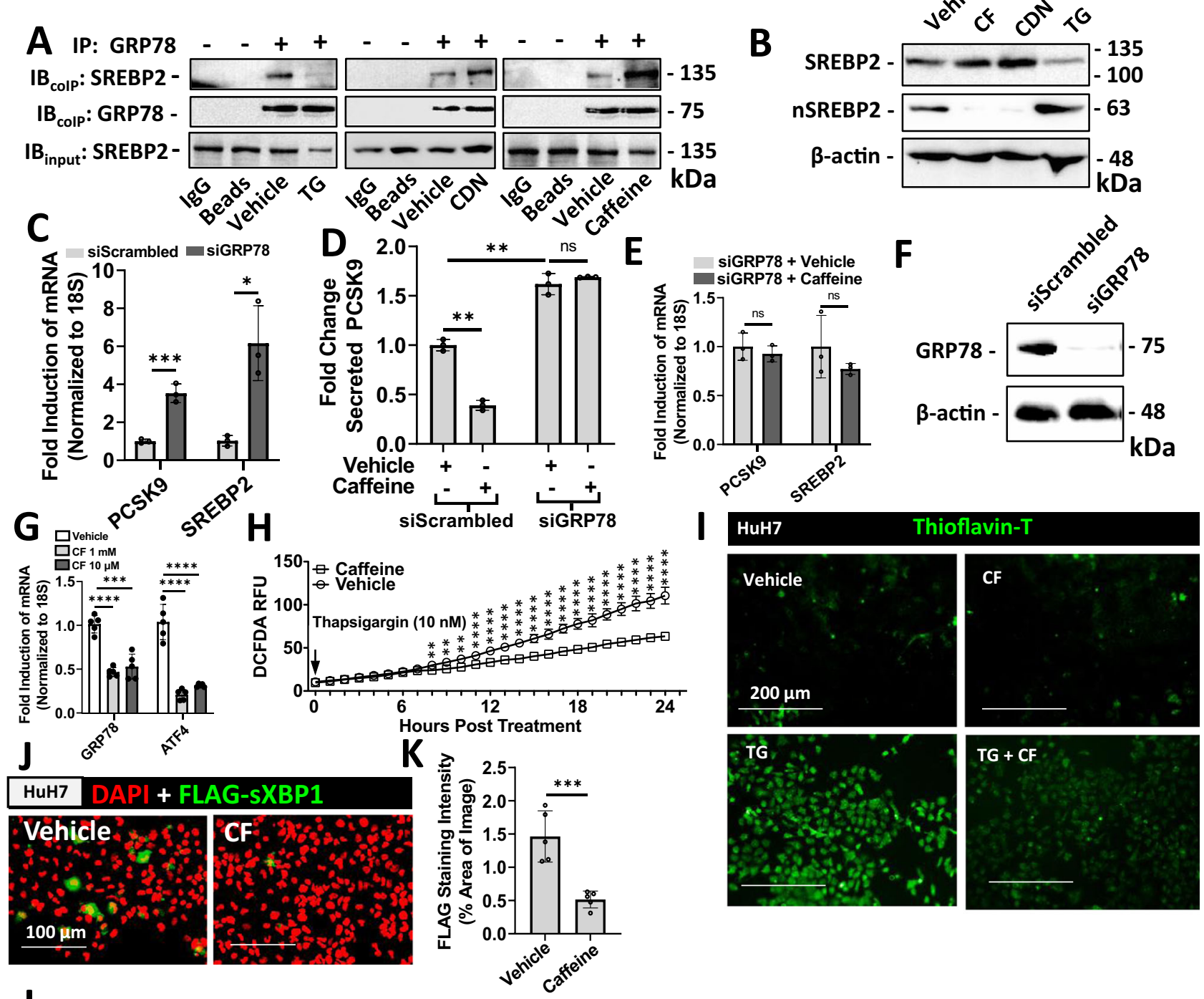

$\mathbf{L}$

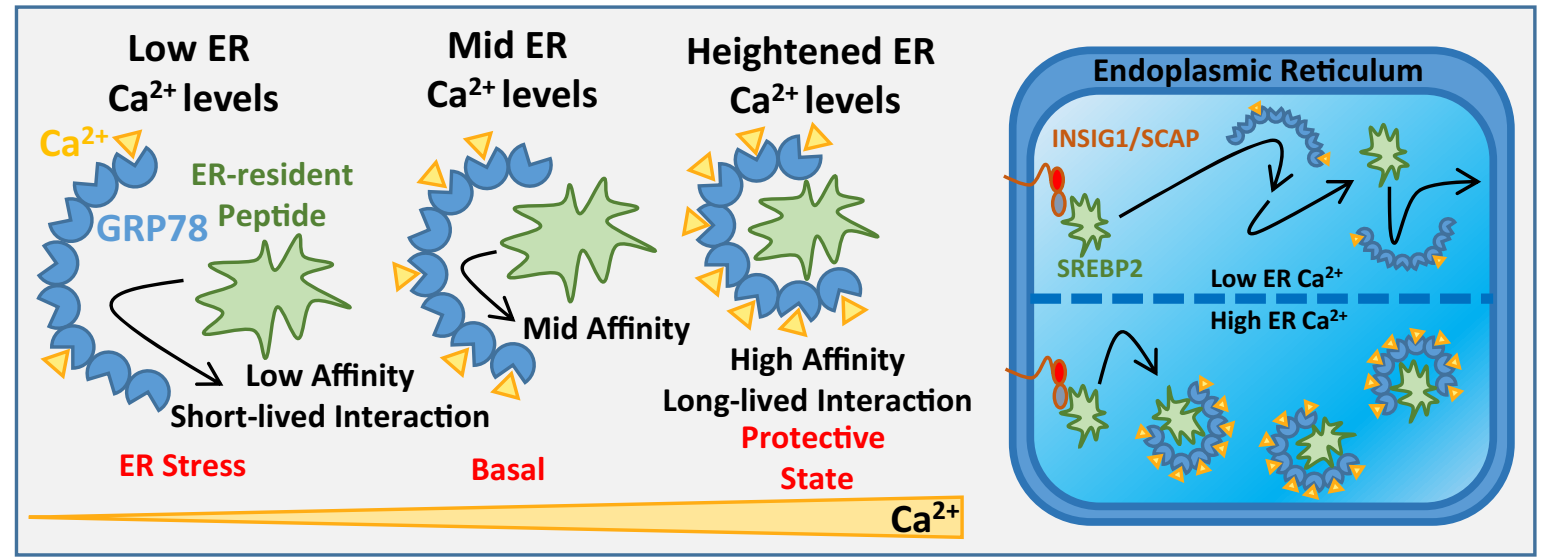

DiI fluorescence intensity in $P c s k 9^{+1+}$ mice but failed to affect these parameters in $P c s k 9^{-1-}$ mice (Fig. 6J). Hepatic cell-surface LDLR immunostaining and DiI-LDL uptake were also examined using a fluorescent microscope (Fig. 6K). Finally, native LDLc was also examined in 18-week-old male C57BL/6J mice treated with CF $(30 \mathrm{mg} / \mathrm{kg}$ ) every $24 \mathrm{~h}$ for 14 days (Fig. 6L, M). Using ELISAs, a reduction of the surrogate marker of LDLc, Apolipoprotein $B$ (ApoB), as well as PCSK9 was observed in response to a daily dose of CF. Collectively, these data suggest that CF increases hepatic LDLc clearance by increasing LDLR expression in a manner dependent on its ability to block PCSK9 secretion from hepatocytes.

CF reduces plasma PCSK9 levels in healthy human subjects. Given that CF is among the most commonly consumed pharmacologically active compounds in the world ${ }^{8}$, we assessed its ability to affect PCSK9 levels in fasted healthy volunteers. Serum was collected prior to, as well as 2 - and 4 -h post CF treatment 
Fig. 4 Endoplasmic reticulum $\mathrm{Ca}^{2+}$ regulates the interaction between GRP78 and SREBP2. A HuH7 cells were treated with control agents thapsigargin (TG; $100 \mathrm{nM}$ ), which causes ER Ca ${ }^{2+}$ depletion, or CDN $(100 \mu \mathrm{M})$, a compound known to increase endoplasmic reticulum (ER) Ca ${ }^{2+}$ levels. The effect of caffeine (CF; $200 \mu \mathrm{M})$ was also assessed. Following $24 \mathrm{~h}$ treatment, a co-immunoprecipitation (IP) for GRP78 was carried out. Protein loading was normalized to GRP78 and relative co-immunoprecipitated SREBP2 was examined via immunoblots (IB). B The effect of CF, CDN, and TG on the retention of ER-resident pre-mature SREBP2, and on the activated nuclear SREBP2 (nSREBP2), was also assessed via IB. (C-E) To confirm the role of GRP78 in CFmediated PCSK9 inhibition, mRNA transcript and secreted protein levels were examined in HepG2 cells exposed to siRNA targeted against GRP78 ( siGRP78) ( $n=3$ biologically independent samples per group; data presented are mean \pm s.d.). F Knockdown of GRP78 was confirmed via IB. G ER stress markers were assessed in primary human hepatocytes (PHH) treated with CF $(200 \mu \mathrm{M})$ and CDN $(10 \mu \mathrm{M})$ via real-time PCR ( $n=5$ biologically independent samples per group; data presented are mean \pm s.d.). $\mathbf{H}$ The effect of $\mathrm{CF}$ on reactive oxygen species production, resulting from the treatment of TG (100 nM), was also assessed in HuH7 cells ( $n=3$ biologically independent samples per group; data presented are mean \pm s.d.). I ER stress-induced amyloid deposition was examined using the fluorescent stain, Thioflavin-T (green color). J, $\mathbf{K}$ HuH7 cells were transfected with the ER activated indicator plasmid encoding an ER stress-inducible FLAG-sXBP1 (green color; $n=5$ biologically independent samples per group; data presented are mean \pm s.d.) Staining intensity was quantified using ImageJ software. L Model in which $\mathrm{Ca}^{2+}$ promotes the GRP78-mediated sequestration of SREBP2 in the ER. Statistical comparisons between two groups were conducted using unpaired two-tailed Student's $t$-tests, while multiple groups were compared using oneway ANOVAs with the Tukey HSD post hoc test $\left({ }^{\star} p<0.05 ;{ }^{\star \star} p<0.01 ;{ }^{\star \star \star} p<0.001 ;{ }^{\star \star \star \star} p<0.0001\right)$. Source data are provided as a Source Data file.

(400 mg orally; $\sim 5 \mathrm{mg} / \mathrm{kg}$ ). Consistent with our observations in cultured hepatocytes and in mice, CF reduced plasma PCSK9 levels in healthy subjects by $25 \%(n=12)$ and $21 \%(n=8)$ at the 2- and 4-h time points, respectively (Fig. 7A, B). Plasma PCSK9 levels were also examined in control subjects that did not consume $\mathrm{CF}$, to verify whether the additional $2 \mathrm{~h}$ of fasting during the course of the experiment would alter PCSK9 levels. No significant difference was observed in this group ( $n=5$; Fig. 7C).

Characterization of CF derivatives as antagonists of PCSK9. Our data demonstrated that CF antagonized secreted PCSK9 levels in pre-clinical models, as well as in humans. Interestingly, previous studies have also characterized small molecules that block PCSK9 ${ }^{38,39}$. CF, however, is a well-characterized compound having several health benefits with few known adverse side effects. Achieving an optimal level of PCSK9 inhibition absent of the neuro-excitatory effect of $\mathrm{CF}$, however, may be a challenge for the long-term clinical application of these findings. To address this concern, a variety of caffeine derivatives were screened as potential alternatives to CF that may achieve significant PCSK9 inhibition while avoiding the undesired neuro-excitatory effect (Fig. 8A, B; see chemical structure of CF derivatives in Supplementary Table 2). Interestingly, CF metabolites including theobromine and paraxanthine, as well as other xanthine-derived compounds, such as PSB603, 8CD and 8CC, exhibited a dosedependent reduction of mRNA expression and secreted levels of PCSK9 (Fig. 8A, B).

We have now initiated a medicinal chemistry program with the aim of developing new xanthine derivatives that are optimized for anti-PCSK9 activity. Here we disclose two new compounds, MLRA-1812 and MLRA-1820, that are a product of this effort (Fig. 8C-G). These compounds have significantly greater efficacy for PCSK9 inhibition than CF. Experiments done in HepG2 cells demonstrate that treatment with MLRA-1812 and MLRA-1820 yielded a two-fold reduction of secreted PCSK9 levels compared to $\mathrm{CF}$ treatment at the same dose (Fig. 8C). Moreover, at a concentration of $100 \mathrm{nM}$, these compounds achieved similar levels of inhibition as CF at a dose of $100 \mu \mathrm{M}$. Assessment at the mRNA level revealed a similar mode of action, whereby antagonism of SREBP2 reduced de novo synthesis of PCSK9 (Fig. 8D). Like CF, we also observed that MLRA-1812 and MLRA-1820 did not exhibit cytotoxic properties on cultured HepG2 cells (Fig. 8E). Next, the impact of MLRA-1812 and MLRA-1820 on DiI-LDL uptake was examined. Consistent with the observed reduction in secreted PCSK9 levels, treatment of HepG2 cells with these compounds led to a significant increase in LDLR expression (Fig. 8F) and DiI-LDL uptake (Fig. 8G). Overall, these data demonstrated that a variety of xanthines exhibit potency for the antagonism of PCSK9 expression. The results of our ongoing comprehensive structure-activity relationship studies will be reported in due course.

\section{Discussion}

The effects of CF on the vascular system and CVD have been examined by others in the past ${ }^{8,9}$. Given that CF consumption occurs primarily in the form of beverages that contain inconsistent doses and that are frequently mixed with adulterants such as dairy and sugar product, results from such studies can be difficult to interpret and often vary. A recent meta-analysis provides an in-depth summary of the current body of literature existing on the effects of $\mathrm{CF}$ consumption on cardiovascular outcomes, including total $\mathrm{CVD}^{9}$. Interestingly, the majority of studies examined, which involved thousands to hundreds of thousands of patients, demonstrated a protective effect of $\mathrm{CF}$ consumption against CVD risk.

$\mathrm{CF}$ is known to exert its effect through a range of molecular targets, including the antagonism of adenosine receptors, GABA receptors, and phosphodiesterase enzymes, as well as inducing intracellular $\mathrm{Ca}^{2+}$ transients by enhancing RyR-mediated calcium-induced calcium release (CICR) ${ }^{11}$. Although the aforementioned interactions do not directly support our observation, in which $\mathrm{CF}$ increased $\mathrm{ER} \mathrm{Ca}^{2+}$ levels, $\mathrm{CF}$ is also known to block $\mathrm{ER} \mathrm{Ca}^{2+}$ release via inhibition of the IP3-receptor ${ }^{40,41}$. In addition, CF was previously shown to bind to hepatic RyR and potentially block RyR-mediated $\mathrm{Ca}^{2+}$ release ${ }^{42}$. Given the broad range of targets known to interact with $\mathrm{CF}$, the identification of exact molecular mechanisms pertaining to its protective effect on the vascular system is challenging. In support of the aforementioned protective effect of CF on CVD risk, CF has been shown to promote vasodilation of the vascular endothelium by means of stimulating $\mathrm{Ca}^{2+}$ release via CICR and leading to the activation of $\mathrm{eNOS}^{34}$. In vascular smooth muscle cells, following CICRinduced vasodilation, $\mathrm{CF}$ also increases intracellular $\mathrm{Ca}^{2+}$ levels by inducing non-selective cation channels at the cell surface ${ }^{43}$ and has been shown to block IP3Rs ${ }^{44}$. These studies, as well as those of others ${ }^{45,46}$, are consistent with our observations that CF increases intracellular $\mathrm{Ca}^{2+}$ levels and attenuates ER stress. Collectively, however, there exists a range of mechanisms by which $\mathrm{CF}$ affects intracellular $\mathrm{Ca}^{2+}$ levels, which tend to differ between tissue and/or cell types. In hepatocytes, our data strongly suggest that CF increases cytosolic and ER $\mathrm{Ca}^{2+}$ levels following a $24 \mathrm{~h}$ exposure.

The ER serves as an important and dynamic $\mathrm{Ca}^{2+}$ reserve, capable of extruding $\mathrm{Ca}^{2+}$ for signaling and/or excitatory purposes and removing excess cytosolic $\mathrm{Ca}^{2+}$ following periods of excitement. The $\mathrm{Ca}^{2+}$ sequestering capacity of the ER, which far 

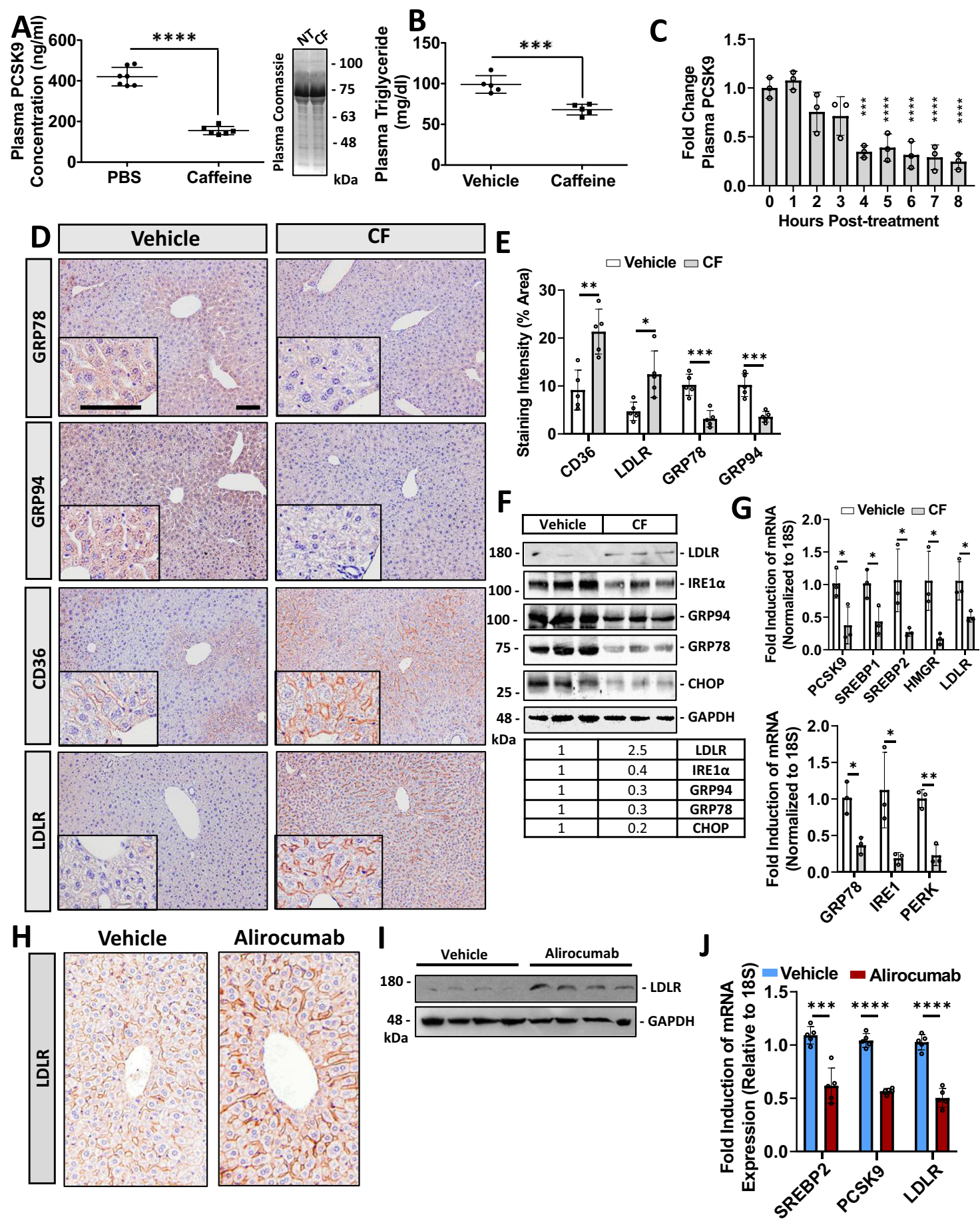

Fig. 5 Caffeine reduces chaperone expression and blocks hepatic PCSK9 expression in mice. 12-week-old male C57BL/6J mice were treated with caffeine (CF; $50 \mathrm{mg} / \mathrm{kg}$ ) and fasted for $8 \mathrm{~h}$ prior to sacrifice $(n=6)$. A, B Plasma PCSK9 and triglyceride levels were measured using an ELISA and colorimetric assays, respectively ( $n=6$ biologically independent samples per group; data presented are mean \pm s.d.). C The time-dependence of CF on plasma PCSK9 levels was also determined using an ELISA ( $n=5$ biologically independent samples per group; data presented are mean \pm s.d.). D The livers of these mice were assessed for cell-surface expression of LDLR and CD36, as well as the ER stress markers GRP78 and GRP94 via immunohistochemical staining $(n=5)$. E Staining was quantified using ImageJ software $(n=5$ biologically independent samples per group; data presented are mean \pm s.d.).

F, G The expression of ER stress markers (GRP78, PERK, and IRE1 $\alpha$ ) as well as cholesterol-regulatory genes (LDLR, PCSK9, HMGR, SREBP1 and SREBP2) were also examined using immunoblots and real-time PCR ( $n=5$ biologically independent samples per group; data presented are mean \pm s.d.). H-I 12week-old male C57BL/6J mice were treated with a single subcutaneous injection of the anti-PCSK9 neutralizing antibody, alirocumab (30 mg/kg), for 10 days $(n=10)$. LDLR expression was assessed using immunohistochemistry and immunoblots. J The mRNA expression of SREBP2, PCSK9, and the LDLR was assessed via real-time PCR $(n=5$ biologically independent samples per group; data presented are mean \pm s.d.). Bars; $50 \mu$ m. Statistical comparisons between two groups were conducted using unpaired two-tailed Student's $t$-tests, while multiple groups were compared using one-way ANOVAs with the Tukey HSD post hoc test $\left({ }^{\star} p<0.05 ;{ }^{* \star} p<0.01 ;{ }^{\star \star \star} p<0.001 ;{ }^{\star \star \star \star} p<0.0001\right)$. Source data are provided as a Source Data file. 

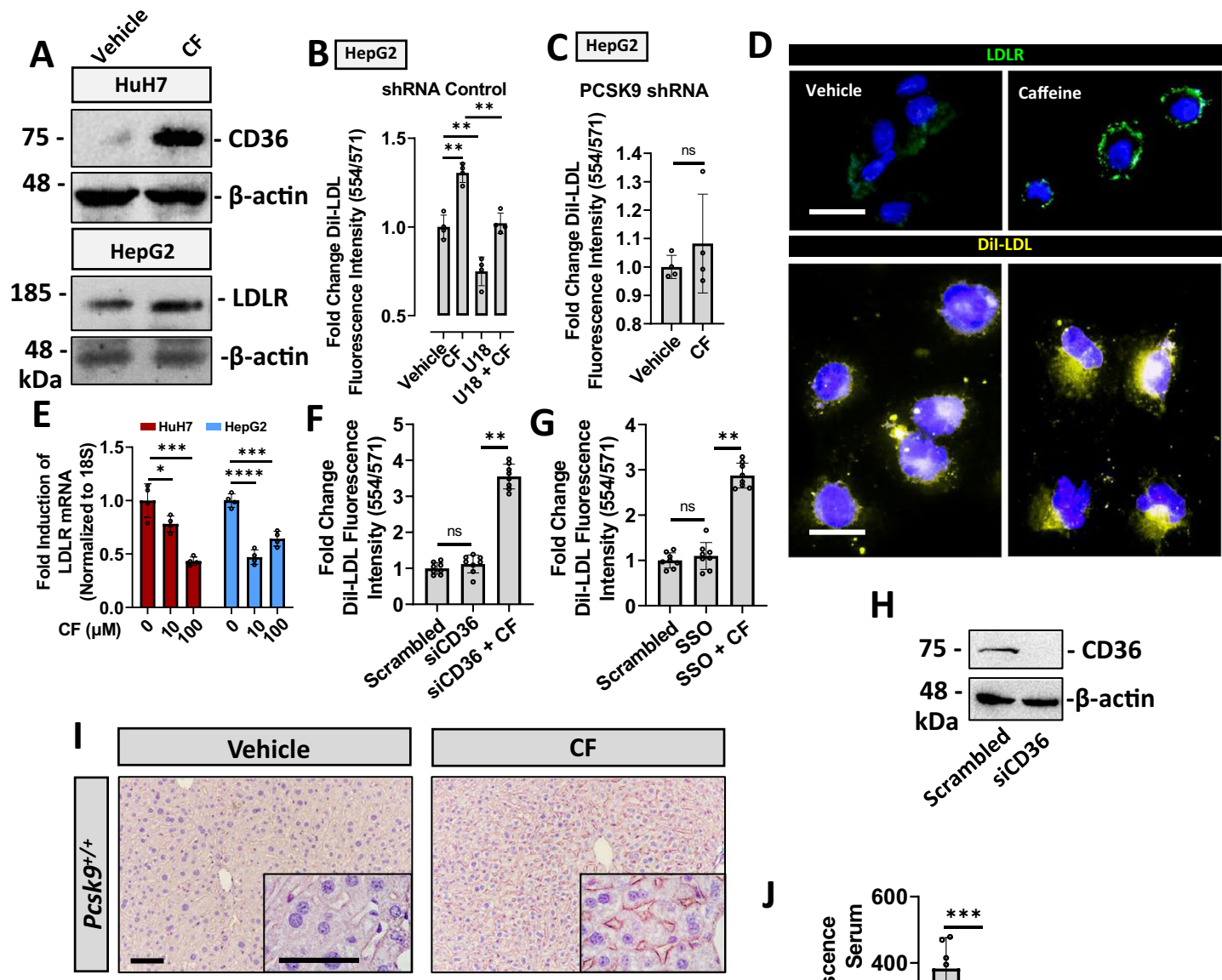

H
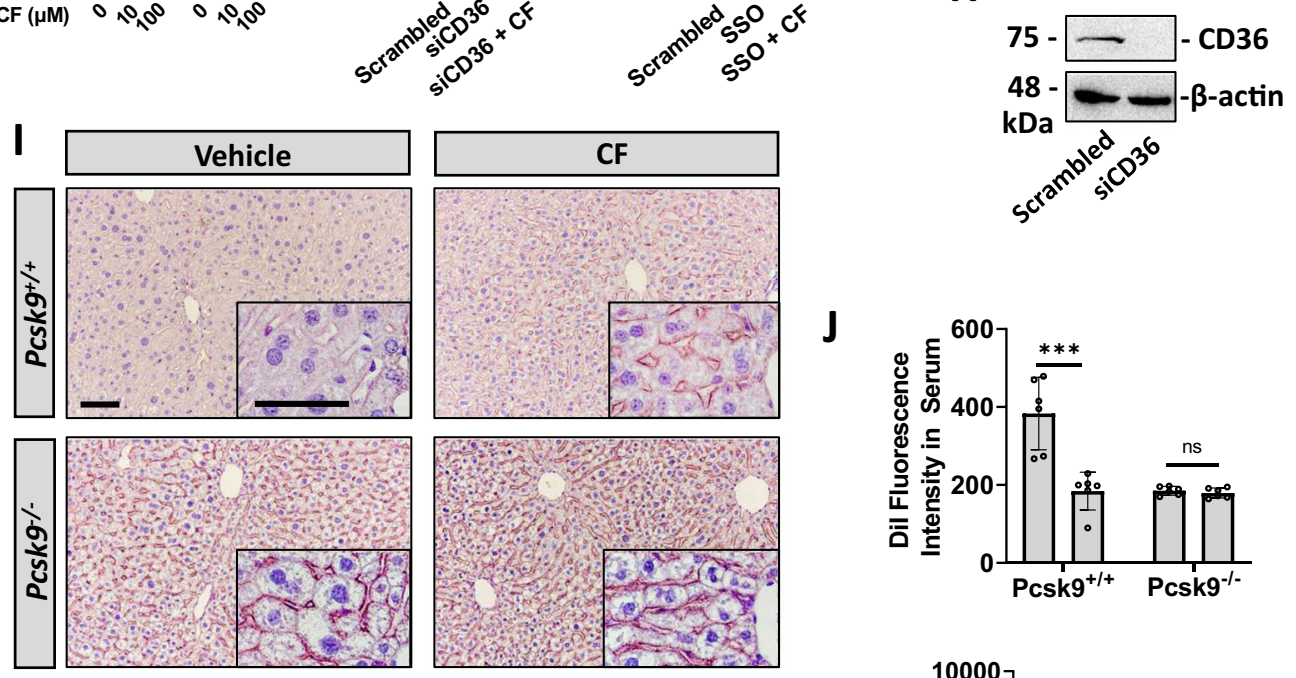

J
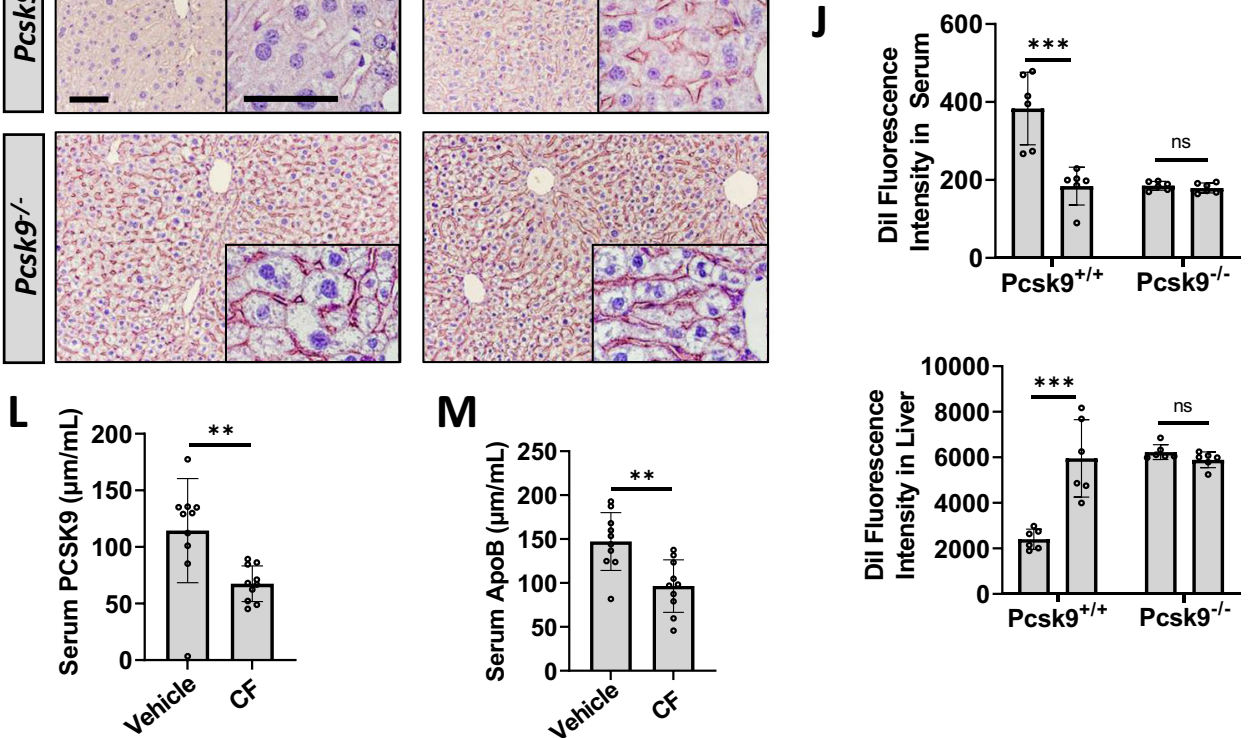

Fig. 6 Caffeine increases hepatic LDL uptake in a PCSK9-dependent manner. A The expression of PCSK9-regulated receptors, LDLR and CD36, was examined in caffeine (CF)-treated cultured hepatocytes $(200 \mu \mathrm{M})$. B The uptake and intracellular accumulation of fluorescently labeled Dil-LDL was examined in cells treated with CF in the presence or absence of the PCSK9-inducer, U18, using a fluorescent spectrophotometer $(n=4$ biologically independent samples per group; data presented are mean \pm s.d.). C The effect of CF treatment $(200 \mu \mathrm{M})$ on Dil-labeled LDL uptake was also examined in PCSK9 shRNA knockdown cells ( $n=4$ biologically independent samples per group; data presented are mean \pm s.d.). D Immunofluorescent staining of cell-surface LDLR was carried out in live CF pre-treated HepG2 cells $(200 \mu \mathrm{M})$. Cellular Dil-LDL accumulation was also visualized in CF-treated HepG2 cells using a fluorescent microscope. E Expression of the LDLR in CF-treated HuH7 and HepG2 cells was measured via real-time PCR $(n=4$ biologically independent samples per group; data presented are mean \pm s.d.). F-G The uptake of Dil-LDL was quantified in HepG2 cells transfected with siRNA targeted against CD36 and a pharmacologic inhibitor of CD36 (SSO) $(10 \mu \mathrm{M})(n=8$ biologically independent samples per group; data presented are mean \pm s.d.). H Knockdown was confirmed via immunoblotting. Pcsk9+/+ and Pcsk9-1- mice were treated with either PBS-vehicle or CF, as well as fluorescently labeled Dil-LDL $(1 \mu \mathrm{\mu g} / \mathrm{kg})$. I-K Hepatic cell-surface LDLR expression was assessed via immunohistochemistry (DAPI: blue; LDLR: green; Dil-LDL: red). J Hepatic and serum Dil-LDL fluorescence intensity was quantified using a fluorescent spectrophotometer and visualized using a fluorescent microscope ( $n=6$ biologically independent samples per group; data presented are mean \pm s.d.). L, $\mathbf{M}$ Native LDLc was also examined in 18-week-old male C57BL/6J mice treated with CF (30 mg/kg) every $24 \mathrm{~h}$ for 14 days via ELISA of the surrogate marker ApoB; serum PCSK9 levels were also assessed via ELISA ( $n=10$ biologically independent samples per group; data presented are mean \pm s.d.). Scale bars; D $10 \mu$ m; I $50 \mu m ; \mathbf{K}$ $100 \mu \mathrm{m}$. Statistical comparisons between two groups were conducted using unpaired two-tailed Student's $t$-tests, while multiple groups were compared using oneway ANOVAs with the Tukey HSD post hoc test $\left({ }^{\star} p<0.05 ;{ }^{\star \star} p<0.01 ;{ }^{\star \star \star} p<0.001 ;{ }^{\star \star \star \star} p<0.0001\right)$. Source data are provided as a Source Data file. 

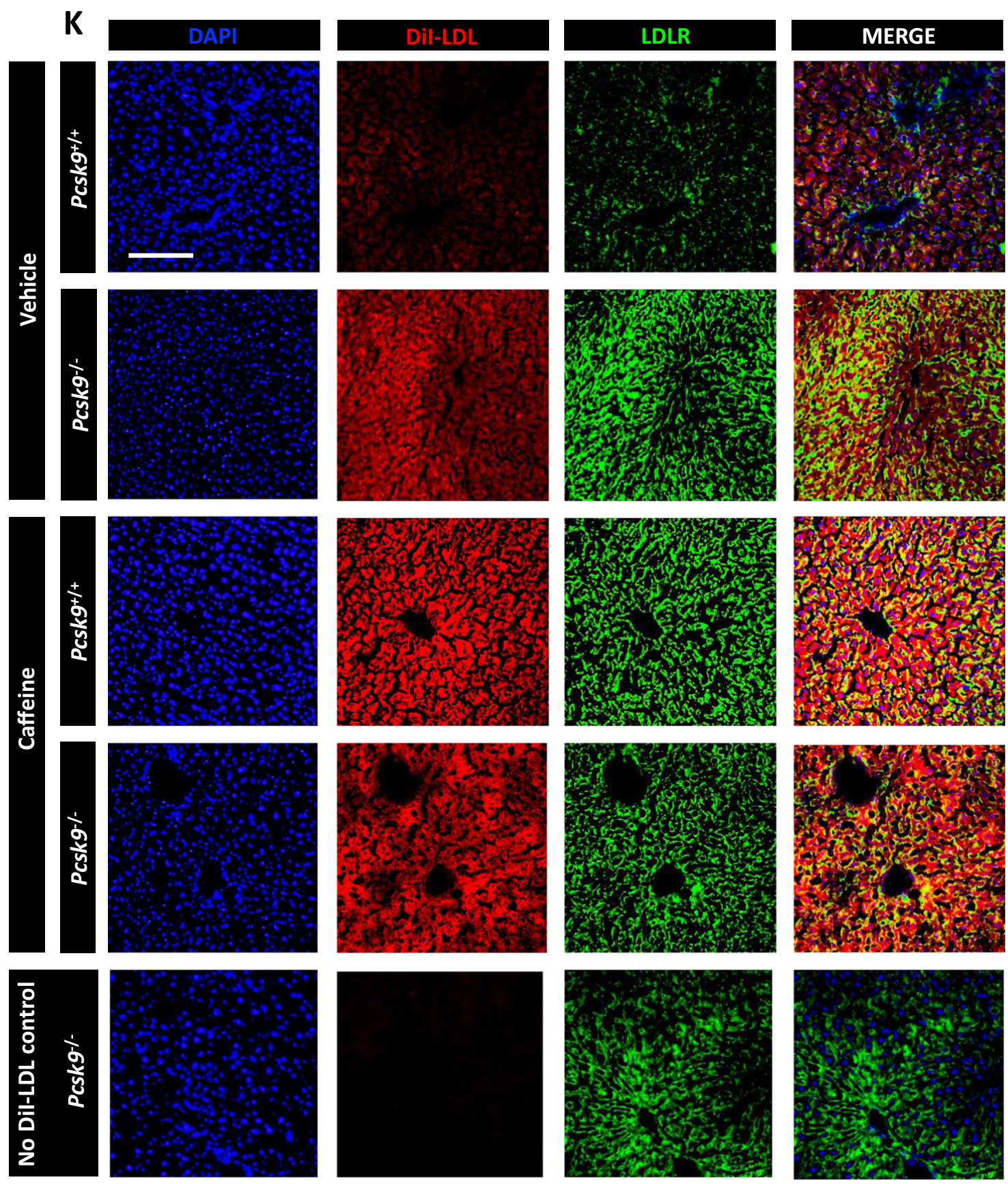

Fig. 6 (Continue)

exceeds that of the cytosol, depends on the abundance of ERresident low-affinity/high capacity $\mathrm{Ca}^{2+}$-binding proteins ${ }^{47}$. The interaction of $\mathrm{Ca}^{2+}$ with these proteins also promotes chaperone function. During conditions of ER $\mathrm{Ca}^{2+}$ depletion, chaperones lose their folding capacity and misfolded polypeptides accumulate in the ER. The UPR, which increases the abundance of ERresident chaperones, is then triggered in order to restore ER folding capacity and $\mathrm{ER} \mathrm{Ca}^{2+}$ levels ${ }^{48}$. Conversely, increasing ER $\mathrm{Ca}^{2+}$ levels appears to have a net protective effect on ER homeostasis, given the observed reduction in UPR marker expression by agents that activate SERCA and increase ER $\mathrm{Ca}^{2+}$ influx or those capable of blocking leakage from either IP3R or RyR $\mathrm{R}^{21,49}$. Consistent with this observation, we also found that CF protected against TG-induced ER stress in cultured hepatocytes and reduced the expression of a variety of ER chaperones in the livers of mice.
In addition to UPR chaperones, ER stress is also known to promote the activation of the self-induced transcription factors that regulate the expression of fatty acid- and cholesterolregulatory genes, namely $S R E B P 1$ and $S R E B P 2^{50}$. Although the exact mechanism by which ER stress activates the SREBPs remains elusive ${ }^{15}$, previous studies have suggested and/or demonstrated that (a) ER stress can reduce the expression of INSIG1, a negative regulator of the SREBPs ${ }^{51}$, (b) ER stressinduced caspases can cleave and activate the SREBPs in a manner independent of conventional S1P activation in the Golgi and ${ }^{52,53}$, and (c) GRP78 can dissociate from the SCAPSREBP complex thus liberating SREBP from the constraints of the $\mathrm{ER}^{54}$. We now report the finding that $\mathrm{ER} \mathrm{Ca}^{2+}$ levels serve to fine-tune the peptide-binding capacity of GRP78, thereby affecting the ER retention of its binding partners, such as pre-mature SREBP2. 

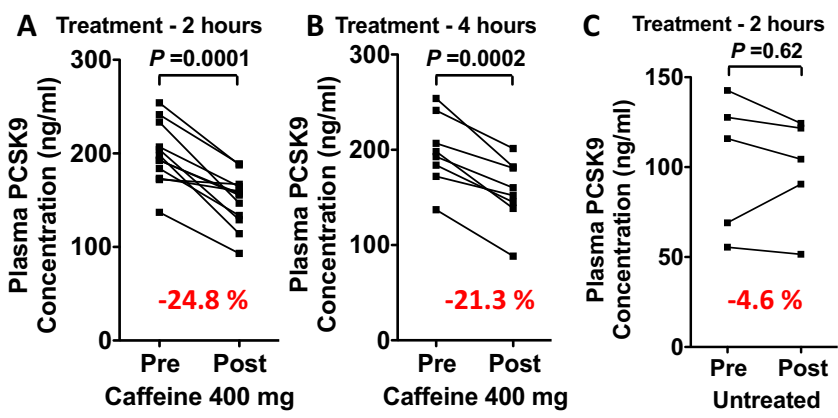

Fig. 7 Caffeine reduces plasma PCSK9 levels in healthy volunteers.

A, B Healthy subjects between the ages of 22 and 45 years were administered $400 \mathrm{mg}(\sim 5 \mathrm{mg} / \mathrm{kg}$ ) of caffeine (CF) following a $12 \mathrm{~h}$ fasting period. Plasma PCSK9 levels were measured before administration, as well as 2- and 4 - $h$ following administration ( $n=12$ and $n=5$, respectively). C PCSK9 levels were also measured in a group of individuals $(n=5)$ that were not administered $\mathrm{CF}$ to control for the additional $2 \mathrm{~h}$ of fasting time during the experiment. Differences between groups were determined using a paired two-tailed Student's t-test.

We also observed that $\mathrm{CF}$ induced the protein expression of the LDLR and increased LDLc uptake in cultured hepatocytes. Given that SREBP2 regulates de novo expression of PCSK9 and the $L D L R$, the observed induction of cell-surface LDLR in the face of SREBP2 inhibition likely occurs in response to the loss of circulating PCSK9 levels. Several studies have demonstrated that, with a half-life of five minutes ${ }^{55}$, PCSK9 expression closely follows that of SREBP2 ${ }^{56}$. In contrast, with a 144 -fold increased half-life compared to PCSK9 $(12 \mathrm{~h})^{57}$, expression of the LDLR appears less dependent on de novo synthesis and more on factors that influence its stability at the cell surface, like circulating PCSK9. Because SREBP2 also induces the expression of HMGR, it is also possible that CF reduces circulating LDLc levels via inhibition of HMGR-mediated de novo cholesterol synthesis. It is well-established, however, that statins block HMGR, induce SREBP2 activity and increase circulating PCSK9 levels. In contrast to statins, our findings demonstrate that CF blocks both SREBP2 and PCSK9. Thus, with reduced circulating PCSK9 levels, it is unlikely that HMGR activity is increased in response to $\mathrm{CF}^{58}$.

PCSK9 enhances degradation of the LDLR and promotes the onset and progression of CVD, which represents one of most challenging and costly health care problems that society faces today $^{59}$. Developing our understanding of the regulatory mechanisms that modulate the expression and secretion of PCSK9 from hepatocytes may aid in the development of antiPCSK9 therapies that are less costly than those that are currently available. Overall, results from our study support a model in which small molecules like $\mathrm{CF}$, capable of increasing $\mathrm{ER} \mathrm{Ca}^{2+}$ levels, can block the activation of SREBP2 by enhancing GRP78 chaperone function and binding capacity (Fig. 9). We also report that CF potently blocks the expression of PCSK9, a downstream target of SREBP2 transcriptional activity, in cultured hepatocytes, in mice, and in healthy human subjects. By extension, we also observed that CF induced the expression of cell-surface hepatic LDLR and increased the uptake of LDLc. Our findings delineate a mechanism by which $\mathrm{ER} \mathrm{Ca}^{2+}$ and its modulators can affect the expression and activity of proteins that play a central role in CVD. Overall, this study provides compelling evidence that the xanthine scaffold is a potent starting point for the development of compounds capable of mitigating CVD risk.

\footnotetext{
Methods

Cell culture, treatments, and transfections. HuH7 (kind gift from Dr. Nabil G. Seidah) and HepG2 (ATCC; HB-8065) cells were routinely grown in complete
}

Dulbecco's Modified Eagle's Medium (Gibco, Thermofisher Scientific) supplemented with $10 \%$ fetal bovine serum (Sigma-Aldrich) and $100 \mathrm{U} / \mathrm{ml}$ of penicillin and streptomycin (Sigma-Aldrich). CF, ryanodine, $2 \mathrm{APB}, \mathrm{CDN}$, theobromine, paraxanthine, 8-cyclopentyl-1,3-dimethylxanthine (8CD), 8-(3-Chlorostyryl) $\mathrm{CF}$ (8CC), PSB603, cyclopiazonic acid and U18666A were purchased from Tocris Bioscience. All cell treatments were carried out for $24 \mathrm{~h}$ unless otherwise stated. Cells were transfected with a cocktail consisting of plasmid DNA $(1 \mu \mathrm{g})$,

X-tremeGENE HP ( $3 \mu \mathrm{l}$; Thermo Fisher Scientific), and opti-MEM (100 $\mu$ l; Thermo Fisher Scientific) per $1 \mathrm{ml}$ complete medium containing plated cells. Human PCSK9 was overexpressed using the bicistronic pIRES-EGFP plasmid; calnexin using the mPA-GFP-N1 plasmid. To attenuate the expression of GRP78 and CD36, siGENOME smartpool siRNA was purchased from GE Dharmacon (M-008198-02 and L-010206-00-0005 respectively) and transfected using lipofectamine RNAiMAX as per the manufacturer's protocol.

\section{$\mathrm{Ca}^{2+}$ studies: fluorogenic dyes and genetically encoded FRET-based sensors} Intracellular $\mathrm{Ca}^{2+}$ in Huh7 and HepG2 cells was measured using a high-affinity $\mathrm{Ca}^{2+}$ indicator, Fura-2-AM (Thermo Fisher Scientific). $\mathrm{ER} \mathrm{Ca}^{2+}$ levels were assessed using the low-affinity $\mathrm{Ca}^{2+}$ indicator, Mag-Fluo-4, and via transfection of cells with D1ER. The D1ER plasmid encodes an ER-resident calcium binding protein linked to a fluorescent protein and increases in fluorescence intensity upon $\mathrm{Ca}^{2+}$ binding ${ }^{7}$. For assessment using indicators, cells were plated in black clearbottom 96-well plates to a confluence of $70-75 \%$ and treated with $\mathrm{Ca}^{2+}$ modulating agents for $24 \mathrm{~h}(n=6)$. Cells were then washed and incubated with Fura-2-AM $(2 \mu \mathrm{M})$ or Mag-Fluo- $4(2 \mu \mathrm{M})$ for $45 \mathrm{~min}$ at $37^{\circ} \mathrm{C}$ in HBSS containing $20 \mathrm{mM}$ HEPES and $2 \%$ pluronic acid v/v (Thermo Fisher Scientific). Fluorescence intensity of intracellular Fura-2-AM was measured at two distinct wavelengths (ex 340/em 515 and ex 380/em 515), following three consecutive washes, to assess bound and unbound states using a SpectraMax GeminiEM fluorescent spectrophotometer (Molecular Devices, Sunnyvale, California, USA). Fluorescence intensity of MagFluo-4 was quantified at a single wavelength (ex 495/em 515). For assessment using D1ER, HuH7 cells were plated in black clear-bottom 96-well plates to a confluence of $70-75 \%$ and transfected $(n=6)$. Twenty-four hours later, cells were treated with $\mathrm{Ca}^{2+}$ modulating agents for an additional $24 \mathrm{~h}$ and quantified (ex 495/em 515) using a SpectraMax GeminiEM fluorescent spectrophotometer.

Immunoblot analysis. Cells were washed in phosphate-buffered saline (PBS), lysed in $4 \mathrm{X}$ SDS-PAGE lysis buffer and separated on $7-10 \%$ polyacrylamide gels in denaturing conditions. Gels were transferred to nitrocellulose membranes using the BioRad mini trans-blot system, blocked in 5\% skim milk in tris-buffered saline (TBS) for $1 \mathrm{~h}$ and incubated in primary antibody overnight for $16 \mathrm{~h}$ at $4{ }^{\circ} \mathrm{C}$. Membranes were then exposed to horse radish peroxidase conjugated secondary antibodies and visualized using EZ-ECL chemiluminescent reagent (Froggabio). Band intensities were quantified using ImageJ software (BioRad) against membranes reprobed for housekeeping proteins. A complete list of antibodies used for immunoblot analysis is presented in Supplementary Table 3 of the supplementary materials.

Immunoprecipitations. Cells were grown in $10 \mathrm{~cm}$ dishes were resuspended in ice cold non-denaturing immunoprecipitation buffer containing $20 \mathrm{mM}$ Tris HCL, $137 \mathrm{mM} \mathrm{NaCl}, 1 \% \mathrm{NP}-40,2 \mathrm{mM}$ EDTA, and protease inhibitors (Roche). Total cell protein was normalized using a protein assay (BioRad) and $1 \mathrm{mg}$ of protein from each sample was incubated with $2 \mu \mathrm{g}$ of capture antibody targeted against GRP78 (Santa Cruz Biotechnology; SC-1050) and rotated on a platform for $24 \mathrm{~h}$ at $4{ }^{\circ} \mathrm{C}$. Following incubation, samples were exposed to $100 \mu \mathrm{l}$ of Protein $\mathrm{G}$ magnetic Surebeads (BioRad) for an additional $2 \mathrm{~h}$ on a rotating platform at $4{ }^{\circ} \mathrm{C}$. Beads conjugated to the anti-GRP78 antibody were subsequently isolated and the remaining sample was collected and labeled "input" for use as controls. The magnetic beads underwent four consecutive washes using the non-denaturing IP buffer and resuspended and boiled in $100 \mu \mathrm{l}$ of $4 \mathrm{X}$ SDS-PAGE sample buffer.

Immunofluorescent Staining. Cells were plated in 4-well chamber slides and incubated in complete DMEM for $24 \mathrm{~h}$ and exposed to treatments $24 \mathrm{~h}$ later for an additional $24 \mathrm{~h}$. Cells were fixed with $4 \%$ paraformaldehyde for 30 minutes and washed with either non-permeabilizing, or permeabilizing PBS containing $0.025 \%$ Triton-X. Cells were then blocked with $1 \%$ bovine serum albumin (BSA) for $30 \mathrm{~min}$ and stained with anti-GFP antibody for $1 \mathrm{~h}$ in PBS containing $1 \%$ BSA. Afterwards, cells were washed and incubated with Alexa 488 fluorescently labeled secondary antibodies (Thermo Fisher Scientific), as well as the DAPI nuclear stain. Slides were then mounted with permafluor and visualized using the EVOS FL color imaging system at either $\times 20$ or $\times 40$ magnification. A complete list of antibodies used for immunofluorescence staining is presented in Supplementary Table 3 of the supplementary materials.

Thioflavin-T staining. Following treatment, live cells were incubated in complete DMEM containing $5 \mu \mathrm{M}$ Thioflavin-T (ThT; Thermo Fisher Scientific) for $15 \mathrm{~min}$. Cells were then fixed in $4 \%$ paraformaldehyde and mounted with permafluor. Fluorescent staining was visualized using the EVOS FL color system at either $\times 20$ or $\times 40$ magnification. 

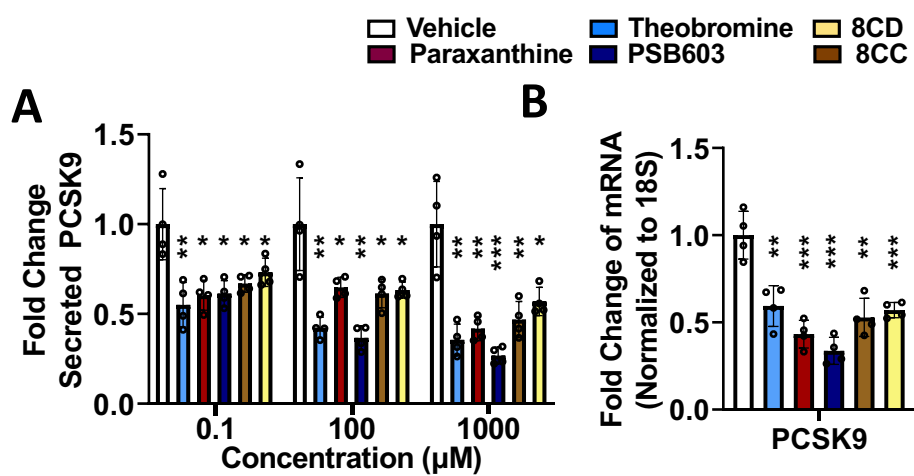

B

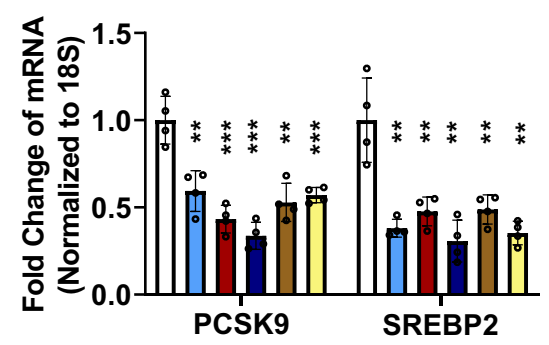

Vehicle $\square 100 \mathrm{nM} \square 100 \mu \mathrm{M}$
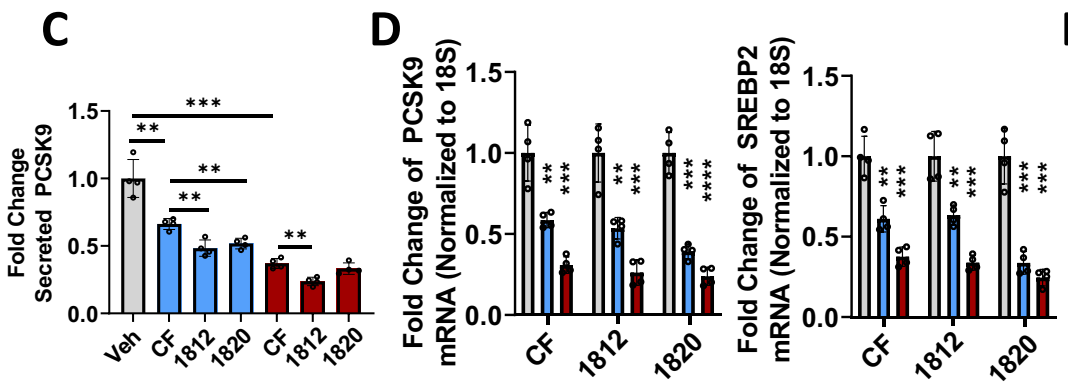

E
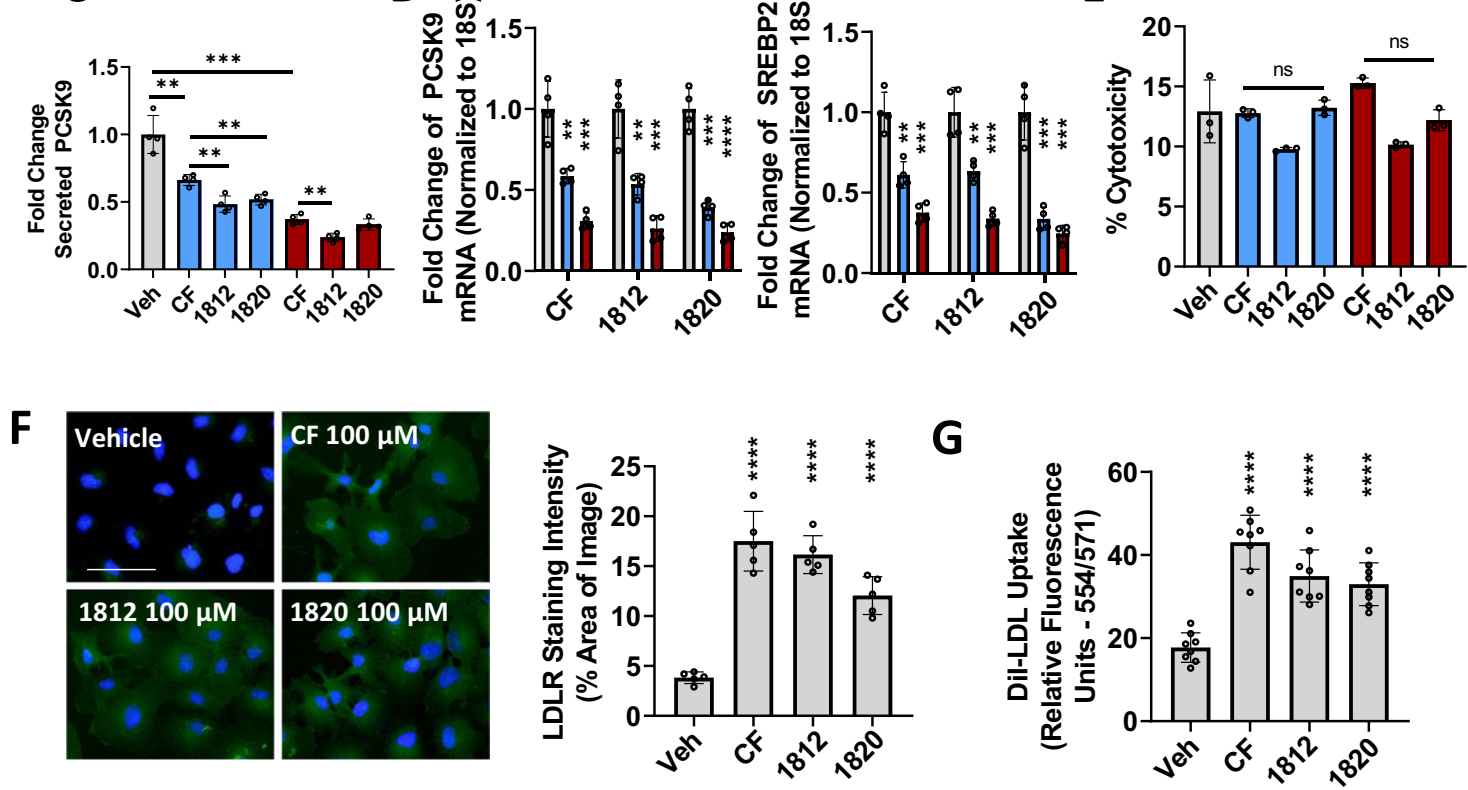

Fig. 8 Characterization of xanthine-derived compounds as PCSK9 inhibitors. A, B HepG2 cells were treated with increasing doses of caffeine (CF) metabolites, paraxanthine, and theobromine, as well as xanthine derivatives PSB603, 8CD, and 8CC. Secreted PCSK9 levels were assessed using ELISAs and mRNA transcript via real-time PCR. C, D Cells were treated with CF, as well as MLRA-1812 and MLRA-1820. Secreted PCSK9, as well as PCSK9 mRNA and SREBP2 mRNA were assessed in these cells. E The cytotoxicity of these agents was examined using an LDH assay. F HepG2 cells were treated with CF, MLRA-1812 $(100 \mu \mathrm{M})$, and MLRA-1820 $(100 \mu \mathrm{M})$ and assessed for cell-surface LDLR expression via immunofluorescent staining (green color); staining intensities were quantified using ImageJ software. G The uptake of Dil-LDL was also quantified in these cells using a spectrophotometer. ${ }^{\star} p<0.05$ vs. vehicle; Statistical comparisons between two groups were conducted using unpaired two-tailed Student's $t$-tests, while multiple groups were compared using one-way ANOVAs with the Tukey HSD post-hoc test $\left({ }^{\star} p<0.05 ;{ }^{\star \star} p<0.01 ;{ }^{\star \star \star} p<0.001 ;{ }^{\star \star \star \star} p<0.0001\right)$. Source data are provided as a Source Data file.

\begin{abstract}
Immunohistochemical staining. Liver tissues were fixed in formaldehyde and subsequently embedded in paraffin for sectioning. Four $\mu \mathrm{M}$ thick sections underwent epitope retrieval and were subsequently stained with primary antibodies for $16 \mathrm{~h}$ at $4{ }^{\circ} \mathrm{C}$. Slides were then exposed to biotin-conjugated secondary antibodies for $45 \mathrm{~min}$ and then streptavidin peroxidase for $10 \mathrm{~min}$. Staining was visualized using the Nova Red HRP Substrate (Vector Laboratories). A complete list of antibodies used for immunohistochemical analysis is presented in Supplementary Table 3 of the Supplementary materials.
\end{abstract}

Quantitative real-time PCR. RNA purification/isolation was performed using RNeasy mini kits (Qiagen) and normalized to $2 \mu \mathrm{g}$ RNA using a NanoDrop spectrophotometer. Samples were then reverse transcribed into cDNA using Superscript Vilo cDNA Synthesis kit (Thermo Fisher Scientific). Real-time PCR was executed with Fast SYBR Green (Thermo Fisher Scientific) using the $\Delta \Delta \mathrm{ct}$ method on the ViiA7 real-time PCR platform (Thermo Fisher Scientific). A complete list of primers used for PCR analysis is presented in Supplementary Table 4 of the supplementary materials.
ELISAs. Secreted PCSK9 levels were assessed directly in cell culture medium of cells grown in FBS-free medium for $24 \mathrm{~h}$ or in the serum isolated from either mice or human subjects. Mouse PCSK9 was measured using the Quantikinine ELISA kit (\#MCP900, R\&D Systems) and human PCSK9 using the PCSK9 Quantikinine ELISA kit (\#DCP900, R\&D Systems). Serum ApoB levels were also quantified using ELISAs (\#DAPB00, R\&D Systems). Serum samples were diluted as per manufacturer's instructions.

Mouse studies and primary hepatocyte isolation. All animal studies were carried out in 12-week-old, 8 h-fasted, male wild-type, $P \operatorname{csk} 9^{-I-}$ or $A m p k \beta 1^{-I-}$ mice on the C57BL/6J background and were performed in strict accordance with the McMaster University animal care guidelines and approved by the McMaster University ethics board. Mice were housed in 12:12 hour light:dark cycles and fed regular chow ad libitum (2918, Envigo). CF $(25-100 \mathrm{mg} / \mathrm{kg}-8 \mathrm{~h})$ and CDN $(50 \mathrm{mg} / \mathrm{kg})$ treatments were administered via intraperitoneal injection unless specified otherwise. Primary mouse hepatocytes were isolated using a two-step process with EGTA (500 mM in HEPES buffer, Sigma Aldrich) and collagenase $(0.05 \%$ in HEPES buffer, Sigma Aldrich) in 12-week-old male mice on the C57BL/6J 


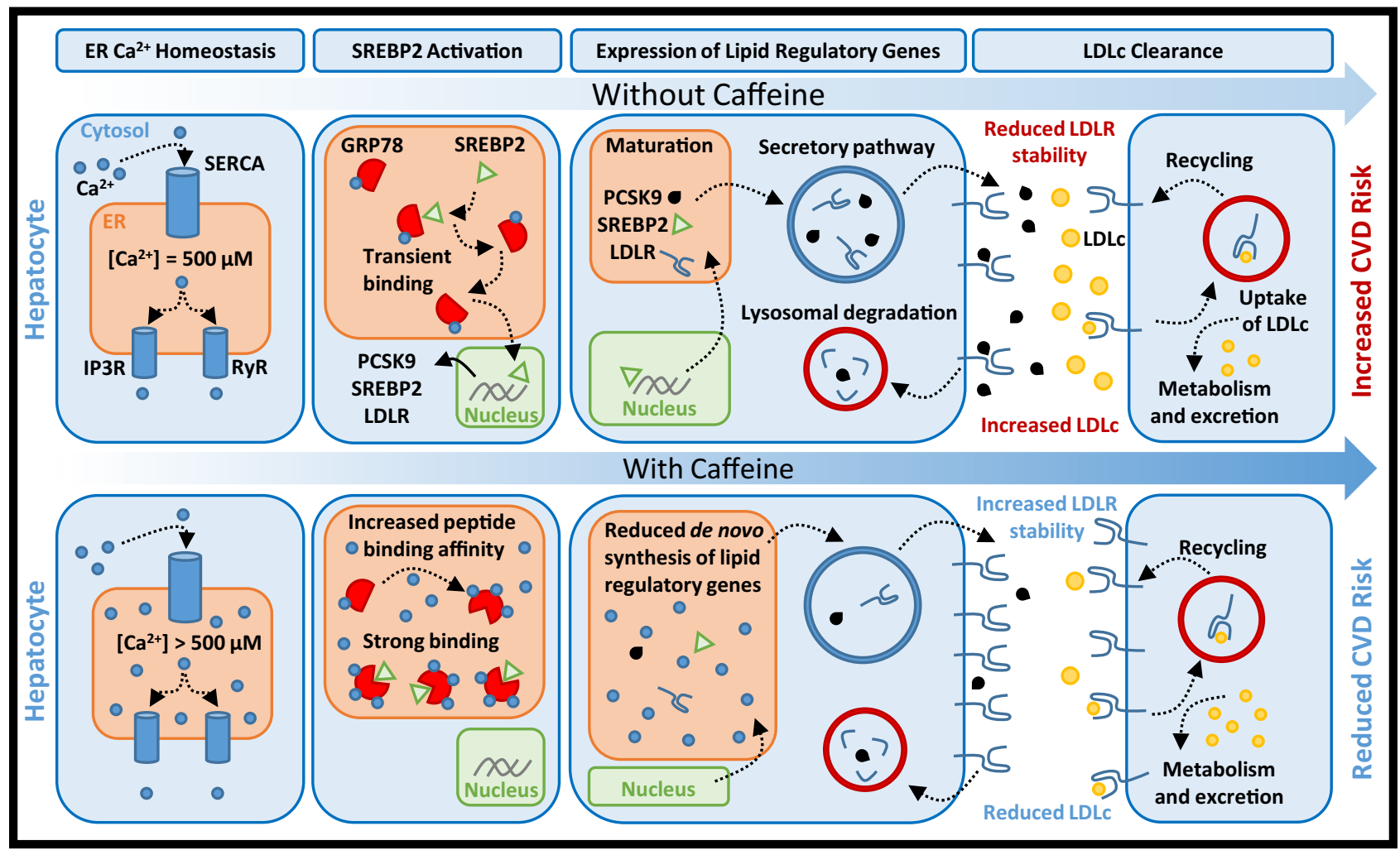

Fig. 9 Caffeine blocks PCSK9 expression and increases LDLc clearance in hepatocytes. The treatment of liver hepatocytes with caffeine increases the concentration of ER Ca ${ }^{2+}$. Excess ER Ca ${ }^{2+}$ leads to an increase in the peptide binding capacity and chaperone activity of ER-resident GRP78. The result is an ER-resident GRP78-SREBP2 complex with enhanced stability. The failure of SREBP2 to quickly exit the ER leads to a net reduction in expression of lipid regulatory genes, including PCSK9, SREBP2 and PCSK9. With reduced outflow of de novo PCSK9, cell-surface LDLR exhibits increased half-life and abundance, leading in a net increase in LDLc clearance.

background. Cells were then washed, centrifuged, and plated following isolation in cell strainers. Hepatosure 100-donor pooled primary human hepatocytes were purchased from Xenotech. Primary hepatocytes were regularly grown in William's E medium (Gibco, Thermo Fisher Scientific) containing 10\% fetal bovine serum, $100 \mathrm{IU} / \mathrm{ml}$ penicillin, and $100 \mu \mathrm{g} / \mathrm{ml}$ streptomycin.

Dil-LDL uptake assay. Cells were seeded plated in black clear-bottom 96-well plates for $24 \mathrm{~h}$ and treated with experimental agents for an additional $24 \mathrm{~h}$. During the last $5 \mathrm{~h}$ of treatment $(19-24 \mathrm{~h})$ cells were exposed to DiI-LDL $(10 \mu \mathrm{g} / \mathrm{ml})$ and then washed with two changes of pre-warmed $\left(37^{\circ} \mathrm{C}\right) \mathrm{HBSS}$ containing $20 \mathrm{mM}$ HEPES prior to analysis. The intracellular fluorescence intensity of DiI was then quantified using the SpectraMax GeminiEM fluorescent spectrophotometer (Molecular Devices; ex 554/em 571).

CF studies in healthy human subjects. Healthy human subjects between the ages of 22 and 45 ( $n=8$ males; $n=4$ females) underwent fasting for $12 \mathrm{~h}$ prior to oral administration of $400 \mathrm{mg}$ CF ( $5 \mathrm{mg} / \mathrm{kg}$, Wake ups). Blood was collected prior to administration and at 2 and $4 \mathrm{~h}$ following administration. All subjects completed and signed consent forms and studies were approved by the Hamilton Integrated Research Ethics Board, project number 5805. The subjects were not compensated for their participation in the study.

Synthesis of MLRA-1812. To a solution of 5,6-diamino-1,3-dimethyluracil (213.0 mg, $1.25 \mathrm{mmol}, 1$ equiv) in to a solution of $\mathrm{MeCN} / \mathrm{H} 2 \mathrm{O}(9: 1,5 \mathrm{~mL})$ were added 3,5-bis(trifluoromethyl)benzaldehyde $(1.25 \mathrm{mmol}, 1$ equiv), azobisisobutyronitrile (AIBN, $4.1 \mathrm{mg}, 0.025 \mathrm{mmol}, 0.02$ equiv), and $\mathrm{n}$-bromosuccinimide (NBS, $155.7 \mathrm{mg}, 0.875 \mathrm{mmol}, 0.7$ equiv). The reaction mixture was stirred at $30^{\circ} \mathrm{C}$ for $3 \mathrm{~h}$. A precipitate was filtered and rinsed ethyl acetate $(2 \times 5 \mathrm{~mL})$ and methanol $(3 \times 5 \mathrm{~mL})$ to obtain the crude product. Chromatography over silica gel using gradient elution with DCM and methanol offered MLWK-1812 (8-(3,5-bis(trifluoromethyl)phenyl)-1,3-dimethyl-3,7-dihydro-1H-purine-2,6-dione, $196.1 \mathrm{mg}$, $0.50 \mathrm{mmol}, 40 \%$ yield) as a white solid. Mp: $329-330$ o C. $1 \mathrm{H} \mathrm{NMR}(700 \mathrm{MHz}$, DMSO-d6): d (ppm) 14.38 (s, br, 1H, NH), $8.76(\mathrm{~s}, 1 \mathrm{H}), 8.24(\mathrm{~s}, 1 \mathrm{H}), 3.53(\mathrm{~s}, 3 \mathrm{H}$, CH3), 3.28 (s, 3H, CH3). 13 C NMR DEPTQ (176 MHz, CDCl3): d (ppm) 154.32, $151.09,148.14,146.17,131.38-130.81,126.39,125.41-120.76, q(J=273.1 \mathrm{~Hz})$,
123.31, 29.86, 27.84. FT-IR (cm-1): 3052.09, 2943.32, 2759.41, 1704.53, 1649.52, 1601.04. LCMS (ESI) m/z: 393.07807 calculated for $([\mathrm{M}+\mathrm{H}]+)$ ); 393.07684 observed. The general procedure for the synthesis of MLRA-1812 was based on Bandyopadhyay et al. ${ }^{60}$.

Synthesis of MLRA-1820. A reaction procedure analogous to that described for MLRA-1812 was followed using 2,4,5-trifluorobenzaldehyde to yield MLRA-1820 (1,3-dimethyl-8-(2,4,5-trifluorophenyl)-3,7-dihydro-1H-purine-2,6-dione,

$117.9 \mathrm{mg}, 0.38 \mathrm{mmol}, 30 \%$ yield) as a light brown solid. Mp: $361-363^{\circ} \mathrm{C} .1 \mathrm{H} \mathrm{NMR}$ (700 MHz, DMSO-d6): $\delta$ (ppm) $13.86(\mathrm{~s}, \mathrm{br}, 1 \mathrm{H}, \mathrm{NH}), 8.05-8.01$ (dd, J = 17.1, $9.0 \mathrm{~Hz}, 1 \mathrm{H}), 7.83-7.79(\mathrm{td}, \mathrm{J}=10.4,6.8 \mathrm{~Hz}, 1 \mathrm{H}), 3.49(\mathrm{~s}, 3 \mathrm{H}, \mathrm{CH} 3), 3.27(\mathrm{~s}, 3 \mathrm{H}$, CH3). FT-IR (cm-1): $3281.65,3039.61,1695.87,1651.37,1602.14$. LCMS (ESI) $m / z$ : 311.07504 calculated for $([\mathrm{M}+\mathrm{H}]+)$; 311.07481 observed. The general procedure for the synthesis of MLRA-1812 was based on Bandyopadhyay et al. ${ }^{60}$.

Statistics. Statistical comparisons between two groups were conducted using unpaired Student's $t$-tests, while comparisons between multiple groups were compared using one-way ANOVAs with the Tukey HSD post-hoc test. The paired Student's $t$-test was used to compared pre- and post-treatment values in human subjects. Differences between groups were considered significant at $p<0.05$ $\left({ }^{*} p<0.05 ; * * p<0.01 ; * * * p<0.001 ; * * * * p<0.0001\right)$ and all values are expressed as mean \pm s.d. Data from studies conducted in cell lines and mice are representative of at least three independent experiments.

Reporting summary. Further information on research design is available in the Nature Research Reporting Summary linked to this article.

\section{Data availability}

All data generated in this study are available within the Article, Supplementary Information and Source Data files. Source data are provided with this paper.

Received: 17 August 2020; Accepted: 5 January 2022;

Published online: 09 February 2022 


\section{References}

1. Horton, J. D. et al. Combined analysis of oligonucleotide microarray data from transgenic and knockout mice identifies direct SREBP target genes. Proc. Natl Acad. Sci. USA 100, 12027-12032 (2003).

2. Abifadel, M. et al. Mutations in PCSK9 cause autosomal dominant hypercholesterolemia. Nat. Genet. 34, 154-156 (2003).

3. Seidah, N. G. et al. The secretory proprotein convertase neural apoptosisregulated convertase 1 (NARC-1): liver regeneration and neuronal differentiation. Proc. Natl Acad. Sci. USA 100, 928-933 (2003).

4. Benjannet, S. et al. NARC-1/PCSK9 and its natural mutants: zymogen cleavage and effects on the low density lipoprotein (LDL) receptor and LDL cholesterol. J. Biol. Chem. 279, 48865-48875 (2004).

5. Maxwell, K. N. \& Breslow, J. L. Adenoviral-mediated expression of Pcsk9 in mice results in a low-density lipoprotein receptor knockout phenotype. Proc. Natl Acad. Sci. USA 101, 7100-7105 (2004).

6. Sabatine, M. S. et al. Investigators, evolocumab and clinical outcomes in patients with cardiovascular disease. N. Engl. J. Med. 376, 1713-1722 (2017).

7. Robinson, J. G. et al. Enhancing the value of PCSK9 monoclonal antibodies by identifying patients most likely to benefit. A consensus statement from the National Lipid Association. J. Clin. Lipido. 4, 525-537 (2019).

8. Turnbull, D., Rodricks, J. V., Mariano, G. F. \& Chowdhury, F. Caffeine and cardiovascular health. Regul. Toxicol. Pharm. 89, 165-185 (2017).

9. Ding, M., Bhupathiraju, S. N., Satija, A., van Dam, R. M. \& Hu, F. B. Longterm coffee consumption and risk of cardiovascular disease: a systematic review and a dose-response meta-analysis of prospective cohort studies. Circulation 129, 643-659 (2014).

10. Zucchi, R. \& Ronca-Testoni, S. The sarcoplasmic reticulum Ca2+ channel/ ryanodine receptor: modulation by endogenous effectors, drugs and disease states. Pharm. Rev. 49, 1-51 (1997).

11. Echeverri, D., Montes, F. R., Cabrera, M., Galan, A. \& Prieto, A. Caffeine's vascular mechanisms of action. Int. J. Vasc. Med. 2010, 834060 (2010).

12. Khazaei, M., Moien-Afshari, F. \& Laher, I. Vascular endothelial function in health and diseases. Pathophysiology 15, 49-67 (2008).

13. Lebeau, P. et al. Endoplasmic reticulum stress and $\mathrm{Ca} 2+$ depletion differentially modulate the sterol regulatory protein PCSK9 to control lipid metabolism. J. Biol. Chem. 292, 1510-1523 (2017).

14. Werstuck, G. H. et al. Homocysteine-induced endoplasmic reticulum stress causes dysregulation of the cholesterol and triglyceride biosynthetic pathways. J. Clin. Invest. 107, 1263-1273 (2001).

15. Colgan, S. M., Tang, D., Werstuck, G. H. \& Austin, R. C. Endoplasmic reticulum stress causes the activation of sterol regulatory element binding protein-2. Int. J. Biochem. Cell Biol. 39, 1843-1851 (2007).

16. Quan, H. Y., Kim, D. Y. \& Chung, S. H. Caffeine attenuates lipid accumulation via activation of AMP-activated protein kinase signaling pathway in HepG2 cells. BMB Rep. 46, 207-212 (2013).

17. Dong, B. et al. Strong induction of PCSK9 gene expression through HNF1alpha and SREBP2: mechanism for the resistance to LDL-cholesterol lowering effect of statins in dyslipidemic hamsters. J. Lipid Res. 51, 1486-1495 (2010).

18. Tsuda, S. et al. Caffeine and contraction synergistically stimulate 5'-AMPactivated protein kinase and insulin-independent glucose transport in rat skeletal muscle. Physiol. Rep. 3, e12592 (2015).

19. Li, Y. et al. AMPK phosphorylates and inhibits SREBP activity to attenuate hepatic steatosis and atherosclerosis in diet-induced insulin-resistant mice. Cell Metab. 13, 376-388 (2011).

20. Dzamko, N. et al. AMPK betal deletion reduces appetite, preventing obesity and hepatic insulin resistance. J. Biol. Chem. 285, 115-122 (2010).

21. Kang, S. et al. Small molecular allosteric activator of the sarco/endoplasmic reticulum Ca2+-ATPase (SERCA) attenuates diabetes and metabolic disorders. J. Biol. Chem. 291, 5185-5198 (2016).

22. Palmer, A. E., Jin, C., Reed, J. C. \& Tsien, R. Y. Bcl-2-mediated alterations in endoplasmic reticulum $\mathrm{Ca} 2+$ analyzed with an improved genetically encoded fluorescent sensor. Proc. Natl Acad. Sci. USA 101, 17404-17409 (2004).

23. Diercks, B. P., Fliegert, R. \& Guse, A. H. Mag-Fluo4 in T cells: imaging of intra-organelle free $\mathrm{Ca}(2+)$ concentrations. Biochim. Biophys. Acta Mol. Cell Res. 1864, 977-986 (2017).

24. Lebeau, P. F., Platko, K., Byun, J. H. \& Austin, R. C. Calcium as a reliable marker for the quantitative assessment of endoplasmic reticulum stress in live cells. J. Biol. Chem. 296, 100779 (2021).

25. Williams, D. B. Beyond lectins: the calnexin/calreticulin chaperone system of the endoplasmic reticulum. J. Cell Sci. 119, 615-623 (2006).

26. Chen, W. et al. The ryanodine receptor store-sensing gate controls $\mathrm{Ca} 2+$ waves and Ca2+-triggered arrhythmias. Nat. Med. 20, 184-192 (2014).

27. Bootman, M. D. et al. 2-aminoethoxydiphenyl borate (2-APB) is a reliable blocker of store-operated $\mathrm{Ca} 2+$ entry but an inconsistent inhibitor of InsP3induced Ca2+ release. FASEB J. 16, 1145-1150 (2002).

28. Wang, W. A. et al. Loss of calreticulin uncovers a critical role for calcium in regulating cellular lipid homeostasis. Sci. Rep. 7, 5941 (2017).
29. Coe, H. \& Michalak, M. Calcium binding chaperones of the endoplasmic reticulum. Gen. Physiol. Biophys. 28, F96-F103 (2009).

30. Lievremont, J. P., Rizzuto, R., Hendershot, L. \& Meldolesi, J. BiP, a major chaperone protein of the endoplasmic reticulum lumen, plays a direct and important role in the storage of the rapidly exchanging pool of Ca2+. J. Biol. Chem. 272, 30873-30879 (1997)

31. Flynn, G. C., Pohl, J., Flocco, M. T. \& Rothman, J. E. Peptide-binding specificity of the molecular chaperone BiP. Nature 353, 726-730 (1991).

32. Blond-Elguindi, S. et al. Affinity panning of a library of peptides displayed on bacteriophages reveals the binding specificity of BiP. Cell 75, 717-728 (1993).

33. Yang, J., Nune, M., Zong, Y., Zhou, L. \& Liu, Q. Close and allosteric opening of the polypeptide-binding site in a human Hsp70 chaperone BiP. Structure 23, 2191-2203 (2015).

34. Hatano, Y., Mizumoto, K., Yoshiyama, T., Yamamoto, M. \& Iranami, H. Endothelium-dependent and -independent vasodilation of isolated rat aorta induced by caffeine. Am. J. Physiol. 269, H1679-H1684 (1995).

35. Demers, A. et al. PCSK9 induces CD36 degradation and affects long-chain fatty acid uptake and triglyceride metabolism in adipocytes and in mouse liver. Arterioscler. Thromb. Vasc. Biol. 35, 2517-2525 (2015).

36. Kuhnast, S. et al. Alirocumab inhibits atherosclerosis, improves the plaque morphology, and enhances the effects of a statin. J. Lipid Res. 55, 2103-2112 (2014).

37. Seidah, N. G., Abifadel, M., Prost, S., Boileau, C. \& Prat, A. The proprotein convertases in hypercholesterolemia and cardiovascular diseases: emphasis on proprotein convertase subtilisin/kexin 9. Pharm. Rev. 69, 33-52 (2017).

38. Lammi, C. et al. Computationally driven structure optimization, synthesis, and biological evaluation of imidazole-based proprotein convertase subtilisin/ kexin 9 (PCSK9) inhibitors. J. Med. Chem. 62, 6163-6174 (2019).

39. Londregan, A. T. et al. Small molecule proprotein convertase subtilisin/kexin type 9 (PCSK9) inhibitors: hit to lead optimization of systemic agents. J. Med. Chem. 61, 5704-5718 (2018).

40. Huang, W. et al. Caffeine protects against experimental acute pancreatitis by inhibition of inositol 1,4,5-trisphosphate receptor-mediated $\mathrm{Ca} 2+$ release. Gut 66, 301-313 (2017).

41. Kang, S. S. et al. Caffeine-mediated inhibition of calcium release channel inositol 1,4,5-trisphosphate receptor subtype 3 blocks glioblastoma invasion and extends survival. Cancer Res. 70, 1173-1183 (2010).

42. Pierobon, N., Renard-Rooney, D., Gaspers, L. \& Thomas, A. Ryanodine receptors in liver. J. Biol. Chem. 45, 34086-34095 (2006).

43. Guerrero, A., Singer, J. J. \& Fay, F. S. Simultaneous measurement of Ca2+ release and influx into smooth muscle cells in response to caffeine. A novel approach for calculating the fraction of current carried by calcium. J. Gen. Physiol. 104, 395-422 (1994).

44. Missiaen, L. et al. Inhibition of inositol trisphosphate-induced calcium release by cyclic ADP-ribose in A7r5 smooth-muscle cells and in 16HBE14obronchial mucosal cells. Biochem. J. 329, 489-495 (1998).

45. Hosoi, T., Toyoda, K., Nakatsu, K. \& Ozawa, K. Caffeine attenuated ER stressinduced leptin resistance in neurons. Neurosci. Lett. 569, 23-26 (2014).

46. Teng, R. J. et al. Attenuation of endoplasmic reticulum stress by caffeine ameliorates hyperoxia-induced lung injury. Am. J. Physiol. Lung Cell Mol. Physiol. 312, L586-L598 (2017).

47. Krebs, J., Agellon, L. B. \& Michalak, M. Ca(2+) homeostasis and endoplasmic reticulum (ER) stress: an integrated view of calcium signaling. Biochem. Biophys. Res. Commun. 460, 114-121 (2015).

48. Gorlach, A., Klappa, P. \& Kietzmann, T. The endoplasmic reticulum: folding, calcium homeostasis, signaling, and redox control. Antioxid. Redox Signal 8, 1391-1418 (2006)

49. Luciani, D. S. et al. Roles of IP3R and RyR Ca2+ channels in endoplasmic reticulum stress and beta-cell death. Diabetes 58, 422-432 (2009).

50. Basseri, S. \& Austin, R. C. ER stress and lipogenesis: a slippery slope toward hepatic steatosis. Dev. Cell 15, 795-796 (2008).

51. Bobrovnikova-Marjon, E. et al. PERK-dependent regulation of lipogenesis during mouse mammary gland development and adipocyte differentiation. Proc. Natl Acad. Sci. USA 105, 16314-16319 (2008).

52. Wang, X. et al. Purification of an interleukin-1 beta converting enzyme-related cysteine protease that cleaves sterol regulatory element-binding proteins between the leucine zipper and transmembrane domains. J. Biol. Chem. 270 18044-18050 (1995)

53. Pai, J. T., Brown, M. S. \& Goldstein, J. L. Purification and cDNA cloning of a second apoptosis-related cysteine protease that cleaves and activates sterol regulatory element binding proteins. Proc. Natl Acad. Sci. USA 93, 5437-5442 (1996).

54. Kammoun, H. L. et al. GRP78 expression inhibits insulin and ER stressinduced SREBP-1c activation and reduces hepatic steatosis in mice. J. Clin. Invest. 119, 1201-1215 (2009).

55. Giunzioni, I. \& Tavori, H. New developments in atherosclerosis: clinical potential of PCSK9 inhibition. Vasc. Health Risk Manag. 11, 493-501 (2015). 
56. Jensen, K. K. et al. Dose-dependent effects of siRNA-mediated inhibition of SCAP on PCSK9, LDLR, and plasma lipids in mouse and rhesus monkey. J. Lipid Res. 57, 2150-2162 (2016).

57. Wang, Y., Huang, Y., Hobbs, H. H. \& Cohen, J. C. Molecular characterization of proprotein convertase subtilisin/kexin type 9-mediated degradation of the LDLR. J. Lipid Res. 53, 1932-1943 (2012).

58. Mayne, J. et al. Plasma PCSK9 levels are significantly modified by statins and fibrates in humans. Lipids Health Dis. 7, 22 (2008).

59. Seidah, N. G. The PCSK9 revolution and the potential of PCSK9-based therapies to reduce LDL-cholesterol. Glob. Cardiol. Sci. Pr. 2017, e201702 (2017).

60. Bandyopadhyay, P., Agrawal, S. K., Sathe, M., Sharma, P. \& Kaushik, M. P. A facile and rapid one-step synthesis of 8-substituted xanthine derivatives via tandem ring closure at room temperature. Tet J. 68, 3822-3827 (2012).

\section{Acknowledgements}

pcDNA-D1ER was a gift from Amy Palmer \& Roger Tsien (Addgene plasmid \# 36325) and mPA-GFP-Calnexin-N-14 was a gift from Michael Davidson (Addgene plasmid \# 57122). This work was supported in part by research grants to R.C.A. from the Heart and Stroke Foundation of Canada (G-13-0003064 and G-15-0009389), the Canadian Institutes of Health Research (FRN173520), to N.G.S. from the Leducq Foundation (13 CVD 03), CIHR Foundation grant (148363) and Canada Research Chair (216684). G.R.S. is supported by a CIHR Foundation Grant (201709FDN-CEBA-116200) and a Tier 1 Canada Research Chair and J. Bruce Duncan Endowed Chair in Metabolic Diseases. Financial support from the Research Institute of St. Joseph's Healthcare Hamilton and Amgen Canada is acknowledged. R.C.A. is a Career Investigator of the Heart and Stroke Foundation of Ontario and holds the Amgen Canada Research Chair in the Division of Nephrology at St. Joseph's Healthcare and McMaster University.

\section{Author contributions}

P.F.L., J.H.B., K.P., and R.C.A. conceived the studies and designed the experiments. In vitro studies were conducted by P.F.L. and J.H.B. In vivo studies were conducted by P.F.L. K.P. and M.E.M. Clinical studies were orchestrated by P.F.L. and J.H.B. C.F. derivatives were designed and developed by P.S., M.S., and J.M. The manuscript was written by P.F.L. and R.C.A. and was revised by J.H.B., K.P., G.P., G.R.S., L.J.J., S.R.W.C., N.G.S., S.A.I., M.A.T., and J.M.

\section{Competing interests}

The authors declare the following competing interests: P.F.L., J.H.B, P.S., M.S., J.M., and R.C.A. have filed an institution-owned patent, entitled: "Compounds for Reducing Cholesterol and Treating Liver and Kidney Disease" (18-069_USProv) that relates to the development of caffeine and methylxanthine derivatives to lower cholesterol. R.C.A, J.M., G.P., and M.A.T. have an equity stake in Systemic Therapeutics. G.P. has received honoraria from Amgen and Sanofi. G.R.S. is a co-founder and shareholder of Espervita Therapeutics, a company developing new medications for liver cancer. McMaster University has received funding from Espervita Therapeutics, Esperion Therapeutics, Poxel Pharmaceuticals and Novo Nordisk for research conducted in the laboratory of G.R.S. G.R.S. has received consulting/speaking fees from Astra Zeneca, Eli Lilly, Esperion Therapeutics, Merck, Poxel Pharmaceuticals and Takeda. M.A.T. is the CEO of Exerkine Corporation and Stayabove Nutrition and is actively evaluating and marketing multinutrient supplements for the treatment of aging, obesity, muscular dystrophy and mitochondrial disease. No other authors declare any competing interests.

\section{Additional information}

Supplementary information The online version contains supplementary material available at https://doi.org/10.1038/s41467-022-28240-9.

Correspondence and requests for materials should be addressed to Richard C. Austin.

Peer review information Nature Communications thanks Huang-Ge Zhang, Julien Diana and the other anonymous reviewer(s) for their contribution to the peer review this work.

Reprints and permission information is available at http://www.nature.com/reprints

Publisher's note Springer Nature remains neutral with regard to jurisdictional claims in published maps and institutional affiliations.

(c) (i) Open Access This article is licensed under a Creative Commons Attribution 4.0 International License, which permits use, sharing, adaptation, distribution and reproduction in any medium or format, as long as you give appropriate credit to the original author(s) and the source, provide a link to the Creative Commons license, and indicate if changes were made. The images or other third party material in this article are included in the article's Creative Commons license, unless indicated otherwise in a credit line to the material. If material is not included in the article's Creative Commons license and your intended use is not permitted by statutory regulation or exceeds the permitted use, you will need to obtain permission directly from the copyright holder. To view a copy of this license, visit http://creativecommons.org/ licenses/by/4.0/.

(C) The Author(s) 2022 